\title{
Impacts of Different Driving Automation Levels on Highway Geometric Design from the Perspective of Trucks
}

\author{
Shuyi Wang $\mathbb{D}^{D}$, Bin Yu $\mathbb{D}^{D}$, Yang Ma $\mathbb{D}^{D}$, Jinzhou Liu $\mathbb{D}^{D}$, and Wen Zhou \\ School of Transportation, Southeast University, Nanjing 211189, China \\ Correspondence should be addressed to Bin Yu; yb@seu.edu.cn
}

Received 19 January 2021; Revised 21 March 2021; Accepted 31 March 2021; Published 14 April 2021

Academic Editor: Maria Castro

Copyright (๑) 2021 Shuyi Wang et al. This is an open access article distributed under the Creative Commons Attribution License, which permits unrestricted use, distribution, and reproduction in any medium, provided the original work is properly cited.

Truck automation is emerging as an innovative technology with benefits in traffic safety and the economy to revolutionize freight traffic. Despite these benefits, the potential negative or positive effects of different driving automation levels (from no automation to full automation) on highway geometry remained to be determined. In this study, differences related to sight distance characteristics among varied automation levels were firstly discussed and calibrated. Then, seven analysis scenarios of typical levels were proposed. Based on each level with tailored characteristics, the current models of geometric design elements including the required stopping sight distance, horizontal sight line offset, and lengths of vertical curves were revised. Finally, impacts of each level on computed values of those elements were evaluated. Results show that high or full driving automation could substantially lower the requirements of geometric design. Active safety systems have a similar role but with less significant effects. Differently, the driver assistance and partial or conditional automation systems put a higher demand on the road geometric design in terms of driving safety. Outcomes of this study can be used to design real-world geometry of dedicated lanes and provide a methodological basis for the operation of different driving automation features.

\section{Introduction}

Over the last decades, rapid innovations of artificial intelligence and sensor technology within the automotive sector have brought about dramatic advances in the field of transportation. Vehicles equipped with active safety or driving automation systems (AVs) have received substantial attention for their potential positive effects on safety, traffic flow, energy-saving, and driver workload [1-4]. Besides, the passenger car, truck with automation (AT) is also the primary representative of AV [5].

Due to the considerable reduction in the cost of labor forces [6] and fuels in freight [7], truck automation is expected to penetrate into the market quicker and is more necessary than automation for passenger cars. Also, dedicated truck lanes on highways proposed by Bucklew [8] can serve as the cradle for ATs. However, the potential influence of ATs on highway geometry seems to have received only limited attention to the best of our knowledge [9-12]. Yet, road transportation, an essential mode of transport, accounts for the largest share of transportation worldwide, and the total length of highways in China reached $4,696,300 \mathrm{~km}$ by the end of 2016 [13]. Even a minor impact on highway geometry can therefore have substantial economic and safety implications. Given the rapid growth of driving automation technology, there is a pressing need to uncover its impact on highway geometry.

The Society of Automotive Engineers (SAE) International [14] has provided a taxonomy with detailed definitions of six driving automation levels (Level $0 \sim$ Level 5 , referred to as $L 0 \sim L 5$ hereafter) from none to full driving automation. These levels can be defined by reference to how strong the automation system intervenes in the dynamic driving tasks (DDT) and/or DDT fallback. Drivers are still responsible for the driving tasks before the system evolves into high or full automation. Although many research projects have alluded to a revolutionary introduction of fully automated driving, the development of driving automation for now is better described as an evolutionary process [15-17]. Previous research has demonstrated that 
automation can take the human driver out of the loop, resulting in deteriorated reactions and performances in the worst-case scenario [18-20], for example, system failures or beyond the operational design domain (ODD).

Consideration of driver performance is widely known to be essential to the proper geometric design of highways. The driving behavior of ATs obviously differs from that of human-driven trucks in response time and environmental sensing mode and thus will certainly affect geometry differently $[9,10]$. To the best of our knowledge, Thomas and Martinez-Perez [11] were among the first to examine the impact of automated vehicles on highway geometry. They designed a series of road-train scenarios based on deployments of the well-known Californian Partners for Advanced Transportation and Technology (PATH) and European Safe Road-trains for the Environment (SARTRE). They carried out a quantitative analysis by assuming a continuum of perception reaction time from $0 \sim 2.5 \mathrm{~s}$ with an interval of $0.5 \mathrm{~s}$. Khoury et al. [12] initially investigated the direct effects of a fully autonomous vehicle fleet on geometric design elements including stopping and decision sight distance, and the length of vertical curves. They all suggested that fully automation systems with the complete elimination of the human driver can achieve significant economic improvements, for e.g., considerable reductions in the length of crest and sag vertical curves. However, little is known about the effects of varied driving automation levels on highway geometric design elements. Given that driving automation systems are becoming more and more important, it is necessary to examine the discrepancy among all automation levels and how this, in turn, affects the driver behavior and highway geometric design, which poses potential safety implications.

Considering that highways in service have saturated available land space, it is uneconomical and impractical to design new geometry for resisting the potential negative effects of AVs [12]. By optimizing sensing solutions or fallback strategies, the negative effects of AVs may decrease if they can detect obstacles in an instant and provide reliable support, for example, instant braking or steering for drivers who cannot take-over promptly. From a technical perspective, when equipped with high-definition maps [21, 22], high-tech perception sensors [23], and reliable planning and control algorithms [24], AVs are capable of automatically dealing with risk circumstances and fully adapting to the current practices of highway geometry.

As it remains unknown when and whether fully automated vehicles will come and how the transitional automation levels will affect the geometric design elements, the objective of this study was to quantify the potential negative or positive effects of segregated driving automation levels on highway geometric design from the perspective of trucks. To this end, consideration was given to distinctions in characteristics in terms of sight distances among varied automation levels. The novel contribution for this study is not the computation model of highway geometric elements itself but rather the methods of including features of all automation levels into the models and new insights into potential impacts from varied automation levels. The remainder of this paper is organized as follows. First, differences related to characteristics of sight distance among varied automation levels are discussed. Then, the influence of each automation level with tailored features on highway geometric design elements is calculated and analyzed. The final section provides conclusions of the work.

\section{Sight-Distance-Related Difference Analysis}

First, $L 0$ defined by SAE International [14], is adjusted for our analysis. Then, anticipated performances of human drivers in different automation levels were investigated by describing their possible involved tasks. Based on that, features with respect to the sight distance among varied driving automation levels were elucidated and calibrated by the scrutiny of existing literature and applications.

2.1. Revised Level 0 (No Driving Automation). Design vehicles, including the passenger car, truck, and bus, in many current highway geometric specifications $[25,26]$ do not take any driving automation systems or active safety systems into account. According to [14], such human-driven conventional vehicles with no driving automation (HVs) are considered as L0. Besides, active safety systems, such as forward collision warning (FCW), that merely provide momentary assistance or intervention to help avoid or mitigate potential collisions instead of performing DDT on a sustained basis are also classified as $L 0$. Numerous studies have been conducted to substantiate that warning alerts can promote quicker brake reaction time [27-29]. For stopping sight distance analysis, performance (e.g., the reaction/response time to an unexpected event) of drivers in the loop is particularly important. We excluded some kinds of active safety systems including active control of the vehicle subsystems (brake, throttle, suspension, etc.), for example, automated emergency braking (AEB), because such systems are primarily focused on improving safety rather than comfort and convenience that are two of important factors in highway geometric design.

Therefore, we divided $L 0$ into two sublevels: $\mathrm{LO}^{-}$and $\mathrm{LO}^{+}$ to denote HVs equipped with neither driving automation nor active safety systems, and HVs only supported by active safety systems, respectively, for difference analysis below.

2.2. Driver Tasks and States from LO to L5. Figure 1 displays all possible tasks of the driver in $L 0 \sim L 5$ automation condition (driver's right side) and general measurements of his/ her state (driver's left side). Workload and situation awareness are regarded as two of the most important human factors that are predictive of performance and safety [30]. It is known that the driving automation level is determined by an allocation of roles in DDT and its fallback performance between the human driver and driving automation systems. According to listed tasks in Figure 1 and role definitions in [14], when driving automation systems (if any) are engaged, drivers: 


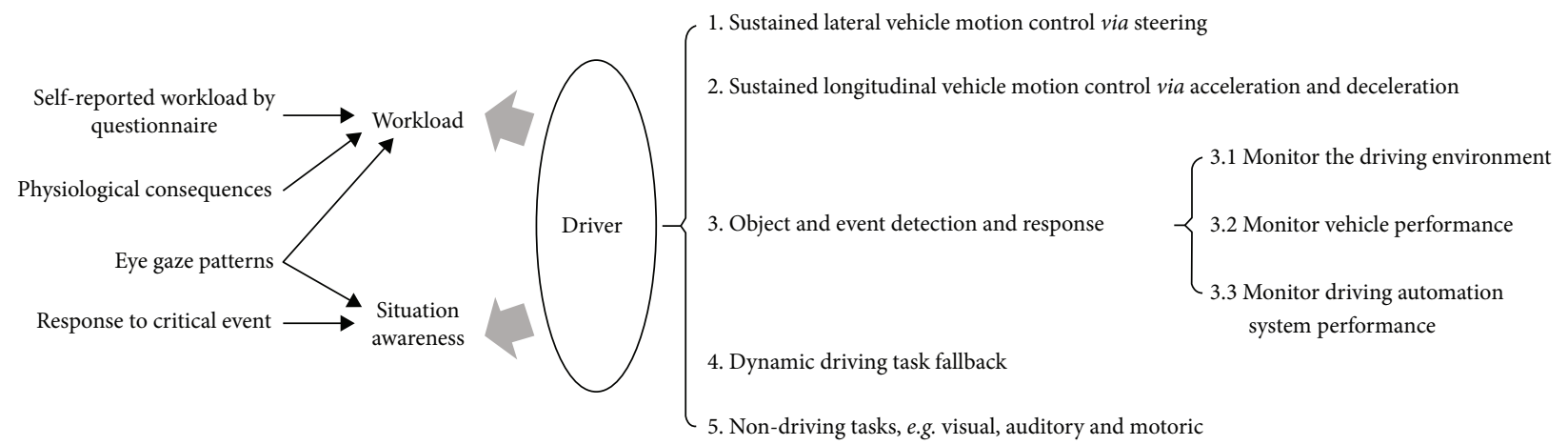

FIGURE 1: Schematic view of the driver's tasks in L0 L5 and measurements of the driver state. Note that (1) nondriving tasks (task 6) do not include those (turning a radio, scanning road signs, answering the phone, etc.) that only require short periods of eyes-off-road time and can be performed simultaneously with driving tasks; (2) strategic tasks (i.e., navigation), such as trip scheduling and route following, were not listed because the difference among automation levels does not include strategic aspects of driving.

(1) In $\mathrm{LO}^{-}$entail no change with those in the conventional vehicle without any automation or active safety systems, i.e., performs task 1 4 except task 3.3

(2) In $\mathrm{LO}^{+}$execute the same tasks as those in $\mathrm{LO}^{-}$but with the support of active safety systems in task 3

(3) In $L 1$ and $L 2$ conduct either task 1 or 2 and neither task 1 nor 2, respectively, and both complete task 3 (with the assistance of driving automation system within its limited dimension, i.e., lateral or/and longitudinal, respectively) and task 4

(4) In $L 3$ are obliged to perform task 4 in a timely manner and are receptive to task 3.2 , and are inclined to pick up task 5

(5) In both $L 4$ and $L 5$ need not perform task 1 4, and are willing to be busy with task 5 as a passenger

Reliefs from the cognitive activity (task 3) and the physical activity (task 1 or/and 2) are associated with a decreased workload [31], and distraction from the road is associated with reduced situation awareness [32]. It is evident that the driving automation system at each level (L1 L5) performs the remainder of tasks (except task 5). Thus, drivers tend to engage in fewer tasks with the evolvement of driving automation. However, fewer engaged tasks do not necessarily cause a reduction in workload and situation awareness. Higher automation $(L 1 \sim L 5)$ could raise workload and strengthen situation awareness with respect to $\mathrm{LO}\left(\mathrm{LO}^{-}\right.$and $\left.\mathrm{LO}^{+}\right)$if the driver has to remain vigilant and monitor/supervise the driving environment (task 3.1) and automation status (task 3.3) [33]. We neglected this divergent result (i.e., higher automation improves the workload and situation awareness) because a wealth of evidence shows that drivers in higher automation condition are more likely to engage in nondriving tasks (task 5) [34] or distraction [35], i.e., higher automation results in lower workload [36] and weaker situation awareness [37]. In addition, results or conclusions of the worst-case scenario at each automation level are the focus in highway geometric study for safety.

It should be noted that although $L 4$ and $L 5$ drivers who can be also considered as passengers perform "poor" in driving tasks, they have already handed over the entire control to automated systems. That means that drivers' performances do not at all negatively impact vehicle's safe driving controlled by reliable systems. Current practical Automated Driving Systems (ADSs, i.e., L3 L5) customarily encompass $\mathrm{LO}^{+}$features. However, what is missing in the literature is the evidence that there is a statistically reliable difference in workload or situation awareness between ADSs with and without the support of $\mathrm{LO}^{+}$features. Hence, we did not take the possible impact of $\mathrm{LO}^{+}$on ADSs into consideration. Lastly, both $L 4$ and $L 5$ drivers/ADSs conduct identical tasks when ADSs are engaged. Differences between $L 4$ and $L 5$ only exist in the requirement of the operational design domain that is beyond the scope of this paper. Thus, we considered these two levels as one $(L 4 / 5)$.

\subsection{Sight-Distance-Related Characteristics of Actors from} LO L5. Actors in driving from L0 L5 involve the human driver and the driving automation system. Their characteristics with respect to sight distance considered in this paper include perception-brake reaction time, the deceleration rate, the height of the actor's sensing unit (i.e., driver's eye and sensors), and the height of the object.

2.3.1. Perception-Brake Reaction Time. In order to interpret the perception-brake reaction time $(P b R T)$ of vehicles equipped with $L 1 \sim L 5$ automation, components of $P b R T$ and the stopping process of each level were analyzed. Figures 2(a) 2(d) depict PbRT components for all levels. Blocks with the same color possess comparable cognitive or physical properties. For example, sensation is defined as the detection of an object above the roadway, and response selection and programming for humans correspond to planning and decision-making for systems. For $\mathrm{LO}^{-}$driver, Green [38] decomposed the human PbRT that served as the gauge into a sequence of components (Figure 2(a)): mental processing time (including subcomponents: sensation, perception and response selection, and programming) and movement time. PbRT for computing the required stopping sight distance usually does not add the last device's (i.e., brake pedal) response time [38]. From the description 


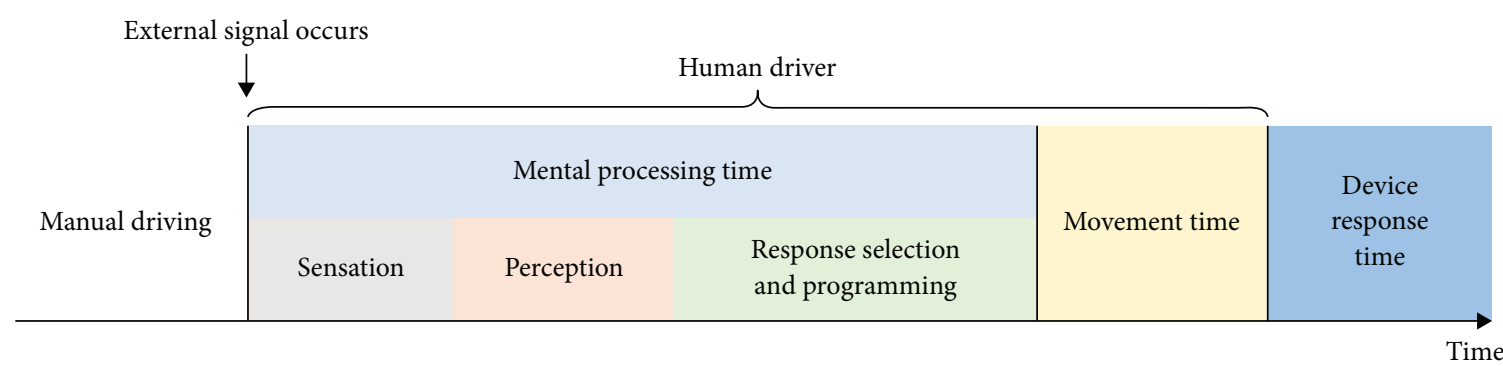

(a)

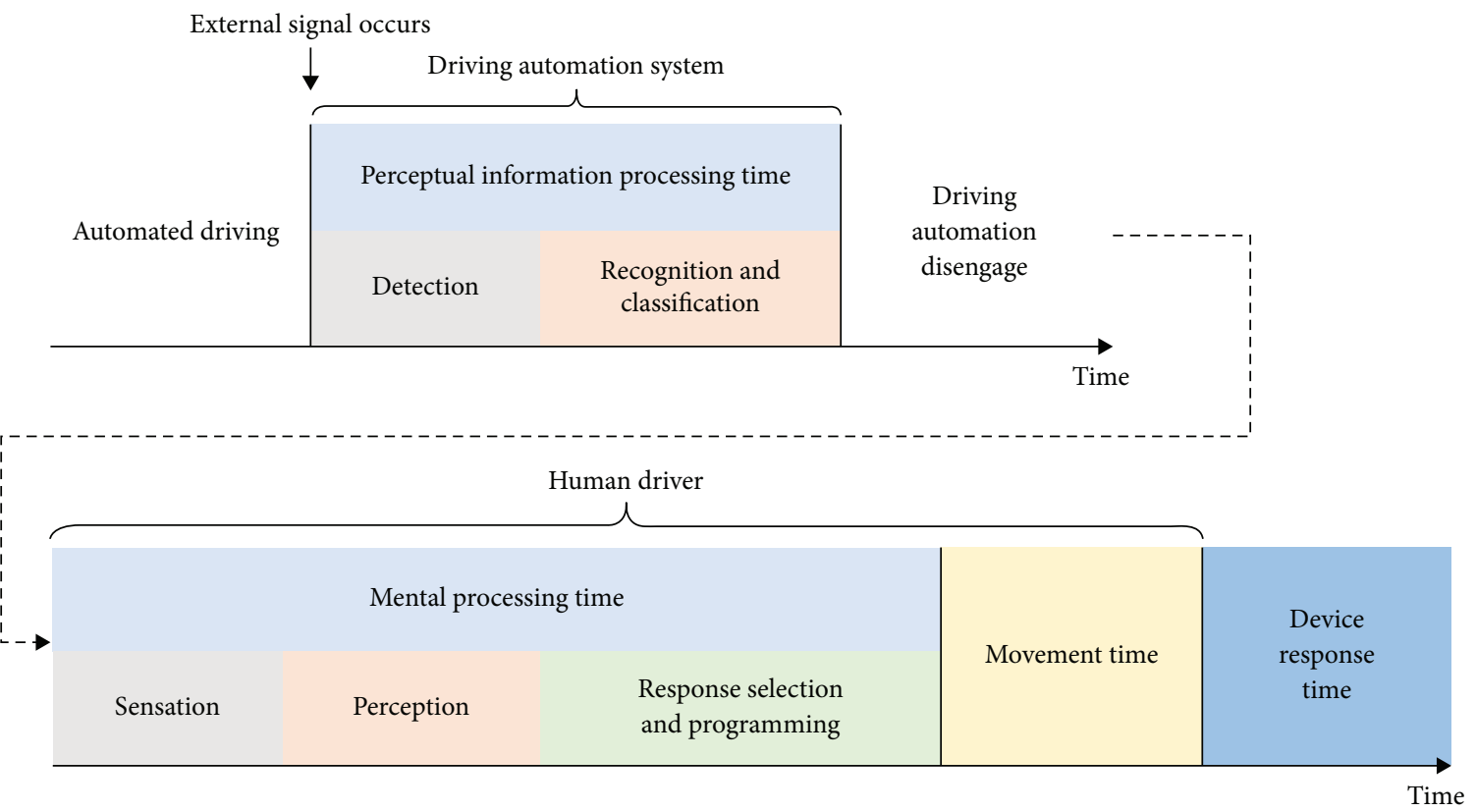

(b)

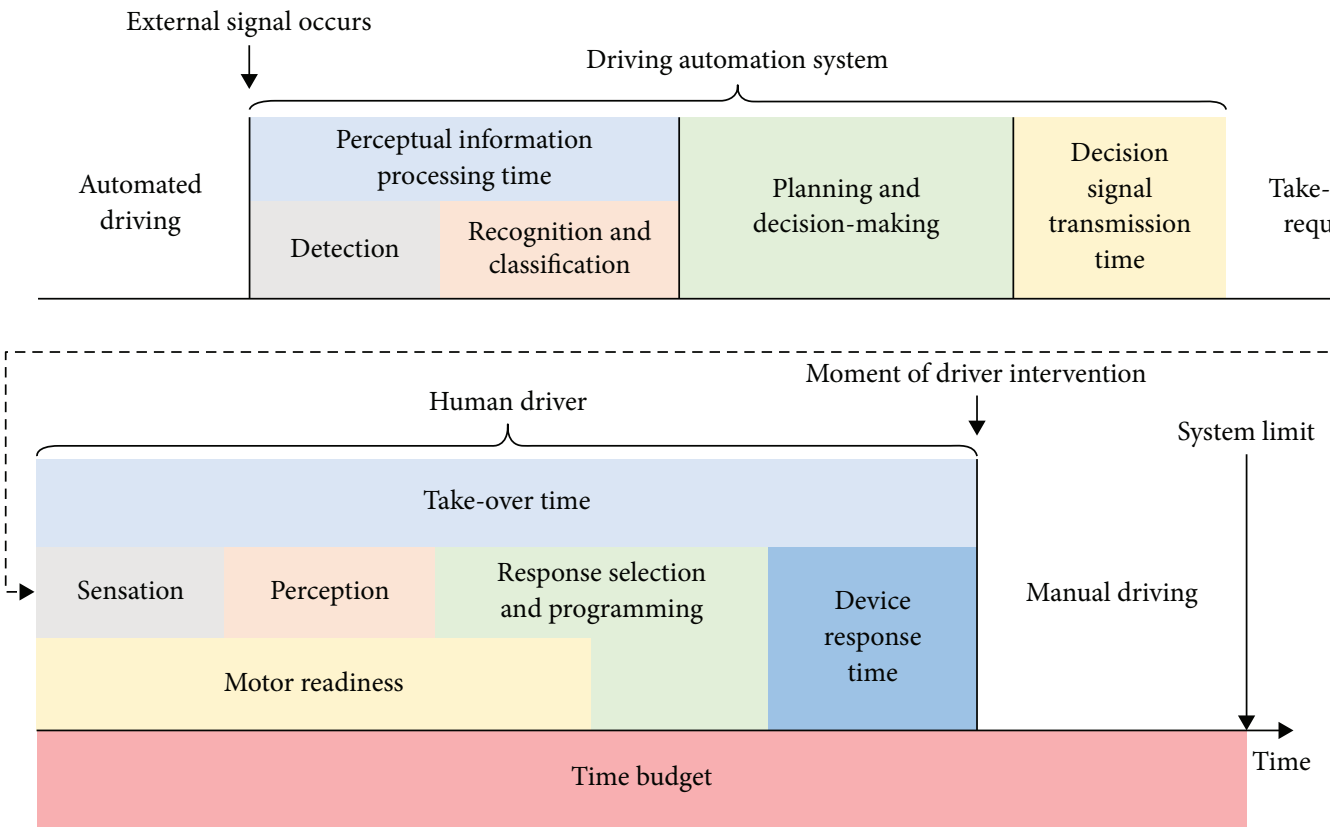

(c)

Figure 2: Continued. 


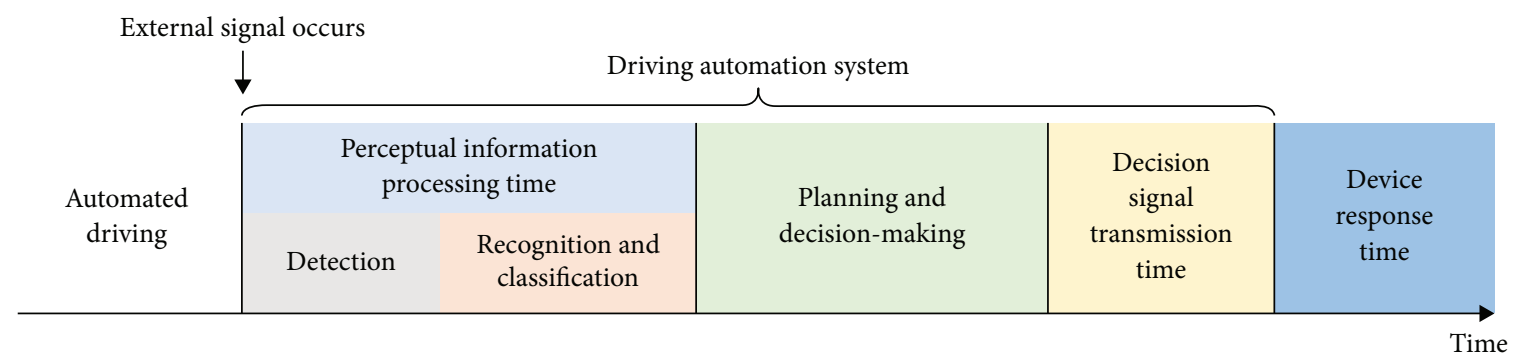

(d)

Figure 2: Decomposed perception-brake reaction time of actors: (a) $L 0^{-}$and $L 0^{+}$; (b) $L 1$ and $L 2$; (c) $L 3$; (d) $L 4$ and $L 5$.

above of drivers' tasks in $\mathrm{LO}^{-}$and $\mathrm{LO}^{+}$, we derived that the $\mathrm{LO}^{+}$ driver maintains the same $P b R T$ components and structure as those of the $\mathrm{LO}^{-}$driver.

For $L 1$ and $L 2$ vehicles, $P b R T$ can be split into two stages: perception reaction time $(P R T)$ of automation system and PbRT of humans (Figure 2(b)). Specifically, PRT of the automation system $\left(P R T_{\text {automation }}\right)$ denotes perceptual information processing time (including subcomponents: detection, recognition, and classification) of $L 1$ or $L 2$ automation. $P b R T$ of human $\left(P b R T_{\text {human }}\right)$ components are the same as those of the $L 0$ driver. It could be possible that $P R T_{\text {automation }}$ and $P b R T_{\text {human }}$ overlap or initiate simultaneously, i.e., the driver determines the disengagement of the system in advance. The worst-case scenario depicted in Figure 2(b) is that $P b R T_{\text {human }}$ occurs after the system disengages. Note that the planning and decision-making block is not included between the sensing and control layers in both $L 1$ and $L 2$ automation control sequence [39] unlike ADS [40] (see Figures 2(c) and 2(d)). Thus, the system withdraws directly when the sensing outcomes exceed the preset threshold.

For $L 3$ vehicles, $P b R T$ consists of two phases: PRT of ADS $\left(P R T_{\mathrm{ADS}}\right)$ and human take-over time (TOT) (Figure 2(c) adapted from Zeeb et al. [41]). In the generic modular system pipeline of ADS [42], if the system determines to issue the take-over request, it routinely occurs after planning and decision-making. Therefore, $P R T_{\mathrm{ADS}}$ covers planning and decision-making, and signal transmission time besides perceptual information-processing time. Components of the take-over process during TOT [41] are basically consistent with those of $P b R T_{\text {human }}$ but with some revisions. Particularly, sensation also includes the detection of the take-over request apart from the object ahead. The driver may simultaneously return his/her hands to the steering wheel and move his/her feet toward the pedal systems to achieve motor readiness [43]. Although the starting moment criteria of driver intervention are ambiguous [44], such as a 2-degree steering angle or $10 \%$ brake pedal position [45], or an absolute steering acceleration of $5 \mathrm{deg} / \mathrm{s}^{2}$ [46], it is believed that the device response time is included. It should be noted that the $L 3$ system will continue to perform DDT fallback with a preemptive brake until the driver intervenes (if any) or the time budget exhausts.

For ADS-dedicated vehicles (i.e., vehicles operated exclusively by L4/5 ADS), PbRT indicates the time it takes for the $L 4 / 5$ system to respond to an external signal. As shown in Figures 2(c) and 2(d), both $P b R T$ for $L 4 / 5$ and $P R T_{\mathrm{ADS}}$ for $L 3$ possess similar components. The former, however, is for the decision to brake whilst the latter is for issuing the request.

2.3.2. Deceleration Rate. The deceleration rate $\left(A_{d}\right)$ elected in measuring braking distance considers approximately 90 percent of all drivers' capabilities to stay within the ego lane and maintain steering control during the braking maneuver on wet surfaces [25]. Explicit in the determination of this $A_{d}$ is the comprehensive assessment of the driving comfort, tirepavement friction level, and capabilities of vehicle braking systems. Notwithstanding the fact that most current vehicle braking systems and friction available on wet pavement surfaces can provide a much larger $A_{d}$, the threshold of that should be determined by the comfort of drivers or passengers, especially in the truck context. Truck drivers may wish to avoid large accelerations, due to trailers risking jackknifing and fragile goods [47]. For L0 L3 vehicles, the human driver still accounts for an appreciable role in DDT or DDT fallback. For L4/5 vehicles, the passenger transformed from driver becomes their important "client." Thus, there is no change in the $A_{d}$ for different levels.

2.3.3. Height of Actor's Sensing Unit. It is known that sight distance is the distance along a roadway throughout which the object is continuously perceptible to the actor. Therefore, the height of the actor's sensing unit and object (see Section 2.3.4) is important for determining sight distance. In $L 0$ condition, the driver's eye is the sensing unit. As described in Section 2.2, the driver still supervises task 3 despite the introduction of $L 1$ or $L 2$ automation. Thus, the driver's eye is the sensing unit in both $L 1$ and $L 2$ automation as before. For ADSs, it is obvious that perception sensors, such as cameras, Radar, and Lidar [48], become the sensing unit instead. If the driver takes over the control of task 1 3 (Figure 1) from ADSs, the human eye becomes the sensing unit again, especially when he/she performs DDT fallback in L3 automation.

For night driving on highways without lighting, the sight distance for $L 0 \sim L 2$ is controlled by the headlight mounting height rather than by the direct line of eye sight. Both Radar and Lidar are independent of light conditions and can work in the darkness [49]. Although many commercial and 
research vehicles utilize multiple identical sensors and varied sensor combinations (i.e., multisensor fusion) to increase perception reliability and robustness, a broad consensus has been reached about utilizing Lidar in the $L 4 / 5$ sensor configuration [50]. For L3 automation, whether Lidar should be installed has not been determined due to current high costs or evolved perception technologies. Tesla, for example, is convinced that vision algorithms realized through cameras enable $L 3$ automation sufficiently [50]. Cameras, however, perform poorly at night.

Furthermore, the upward divergence angle of the sensing beam from the longitudinal axis of the vehicle should be also tailored to the characteristics of the sensing unit at each level. Particularly, it remains the upward divergence angle of the light beam in the $L 0 \sim L 2$ condition. Then, it alters to the upward vertical field of view (FoV) of sensors [51] for ADSs.

2.3.4. Height of Object. The height of object $\left(h_{o}\right)$ is considered as the representative of the smallest or the shortest object that involves risk to human drivers. Driving automation systems are widely known to be greatly beneficial for preventing accidents caused by human errors [52]. It should be noted that although it is difficult to obtain detailed shape information of distant objects by active sensors (e.g., Lidar and Radar) with the limited vertical resolution [53] (i.e., the obtained point cloud is sparse), recognition using camera images enables to perceive small distant objects successfully. However, to the best of our knowledge, no systematic investigation has studied the possible $h_{o}$ resulting in AV crashes. It is anticipated that the $h_{o}$ for driving automation systems sufficient to provide a safety margin against accidents would increase to a certain extent. Because AVs with dedicated sensors will have an enhanced capability to perceive situations involving collision risk within the perception range, it is anticipated that the $h_{o}$ for driving automation systems sufficient to provide a safety margin against accidents would increase to a certain extent.

2.4. Design Criteria for all Automation Levels. For a general analysis, two much-cited design specifications of highway alignment $[25,26]$ were selected as the basic reference for the design criteria of $\mathrm{LO}^{-}$.

2.4.1. Perception-Brake Reaction Time. Although multiple studies have stated that there is no such thing as a single, general $P b R T$ for all drivers $[38,41]$, we adopted the relatively valid fixed $P b R T$ tailored for each automation level. Because sufficient convergence exists among studies of the similar object and methodology to enable reasonable estimates, situation awareness can be inferred from a driver's response to an unexpected event (Figure 1). As a result, the anticipated trend of $P b R T$ results is in the same direction to that of situation awareness (see Section 2.2). Green's much-cited review [38] declared that the $P b R T$ of 2.50 s adopted in [25] is a reasonable guess for the $90 \%$ to $95 \%$ general population. Predominantly driven by a trained group of professionals, as opposed to occasional drivers in the car context, truck drivers will response or take back control quicker than passenger car drivers [54]. Because the separate $P b R T$ for trucks and passenger drivers is not generally used in highway design [25], the $P b R T$ of truck drivers in $\mathrm{LO}^{-}$is still $2.50 \mathrm{~s}$. Bao et al. [55] investigated how a crash warning system influences truck drivers' brake reaction time (i.e., $P b R T$ ). Shorter $P b R T$ $($ mean $=1.62 \mathrm{~s})$ was observed in drivers with alert signals, which correspond to the PbRT (1.64 s) for alerted drivers in [25]. Accordingly, we adopted the PbRT of $1.62 \mathrm{~s}$ in $\mathrm{LO}^{+}$.

Onboard vehicle detection systems have high computational requirements as they need to process the acquired sensing data in real-time for driver/system reactions. For nontrivial velocities of AVs, processing latency should be no larger than $100 \mathrm{~ms}$ typically [56]. Thus, the $P R T_{\text {automation }}$ $0.10 \mathrm{~s}$. Young and Stanton [57] investigated $P b R T_{\text {human }}$ (skilled drivers) under $L 1$ and $L 2$ automation by driving simulator experiments. The authors reported that the mean $P b R T_{\text {human }}$ of $L 1$ and $L 2$ to unexpected events is $3.17 \mathrm{~s}$ (standard deviation $[\mathrm{sd}]=0.289 \mathrm{~s})$ and $3.63 \mathrm{~s}(\mathrm{sd}=0.354 \mathrm{~s})$, respectively. However, recent studies [58-60] have found that $L 2$ automation does not worsen the brake reaction time on a statistically significant level. Naujoks et al. [61] explained that only partial automation (L2) with the instruction to keep the hands off the steering wheel leads to an increase in brake reaction times. It is important to emphasize that $L 2$ usually allows hands-free driving only for a limited amount of time $[14,62]$. Therefore, under the assumption of the normally distributed sample, we estimated that the $90^{\text {th }} P b R T_{\text {human }}$ in both $L 1$ and $L 2$ adopts the same value of $3.50 \mathrm{~s}$. Thus, the PbRT in both $L 1$ and $L 2$ is $3.60 \mathrm{~s}$.

The $P R T_{\mathrm{ADS}}$ was found reasonably to be in the order of $0.50 \mathrm{~s}$ [12], larger than the $P R T_{\text {automation }}$ of $0.10 \mathrm{~s}$ due to the additional work of planning and decision-making. DinparastDjadid [63] reviewed and investigated effects of many important factors (e.g., the time budget to collision, takeover event types) on drivers' $85^{\text {th }}$ percentile TOT value, which is an important performance metric. The authors reported that the mean and $85^{\text {th }}$ percentile TOT for the revealed stopped vehicle event is $2.34 \mathrm{~s}(\mathrm{sd}=1.27 \mathrm{~s})$ and $3.72 \mathrm{~s}$, respectively. We estimated the $90^{\text {th }}$ TOT of $3.80 \mathrm{~s}$. The $P b R T$ in $L 3$ is the gross value of $P R T_{\mathrm{ADS}}$ and TOT. Consequently, the $\mathrm{PbRT}$ inL3 is $4.30 \mathrm{~s}$, while the $\mathrm{PbRT}$ in $L 4 / 5$ remains the same value of $0.50 \mathrm{~s}$ as the PRTADS.

2.4.2. Deceleration Rate. As described in Section 2.3.2, $A_{d}$ of all automation levels remains at its value of $3.4 \mathrm{~m} / \mathrm{s}^{2}$ in [25]. In addition, the $L 3$ system usually started a preemptive braking with a conservative $A_{d}$ of $2.5 \mathrm{~m} / \mathrm{s}^{2}$ during the TOT.

2.4.3. Height of the Actor's Sensing Unit. For large trucks, the recommended value of driver eye height and headlight mounting height for design are $2.33 \mathrm{~m}$ and $1.0 \mathrm{~m}$ [64], respectively, and the upward divergence angle of the light beam is $1 \mathrm{deg}$ [25]. The typical placements of perception sensors on AVs are as follows: (1) forward-facing camera is located either beside the rear view mirror $[49,65]$ or on the vehicle roof [66]; (2) Radar is installed either behind the front bumpers or on the grille $[67,68]$; (3) Lidar is mounted either on the roof (long range) [49] or under the grille (short 
or midrange) [65]. Based on the analysis in Section 2.3.3, the height of the actor's sensing unit $\left(h_{s}\right)$ for $L 0 \sim L 2$ and $L 3$ (during the driver fallback) remains at $2.33 \mathrm{~m}$ in the daytime and $1.0 \mathrm{~m}$ (with $1 \mathrm{deg}$ upward divergence angle of the light beam) at night.

For L4/5 ADSs, the highest mounting height of sensors was selected as $h_{s}$. Thus, without the consideration of the sensor support height, we assigned the truck height as the mounting height of Lidar, i.e., the conservative $h_{s}$ for $L 4 / 5$ is $2.72 \mathrm{~m}$ [64]. Currently, Lidars integrate from 4 to 128 lasers or channels with a vertical FoV that oscillates between 20 and 45 deg [51]. Alexander et al. [69] conducted the attribute analysis of ten Lidar products of several manufacturers (e.g., Velodyne, Hesai, Ouster, and RoboSense), and presented the general specifications of each tested Lidar. Based on those studies, we selected the typical Lidar vertical FoV of 40 deg with an upward divergence of $15 \mathrm{deg}$. For L3 ADSs without the installation of Lidars, both the mounting height of camera and Radar was considered. The generic vertical FoV of long-range Radar proposed in [70] is $5 \mathrm{deg}$. Thus, $2.50 \mathrm{~m}$ [11] (camera mounting height) and $1.0 \mathrm{~m}$ (radar mounting height) with $5 / 2=2.5 \mathrm{deg}$ upward divergence were adopted as $h_{s}$. It should be noted that human engagement in DDT fallback is still the critical scenario in $L 3$ automation (further discussions in Section 3.1).

2.4.4. Height of Object. An object height of $0.60 \mathrm{~m}$ for $\mathrm{LO}^{-}$is representative of the height of passenger car headlights and taillights [25]. Due to very few findings related to $h_{o}$ in the context of driving automation, we assumed a minimum conservative $h_{o}$ of $0.6 \mathrm{~m}$ for all levels.

\section{Methods}

The methodology involved revising current equations of related geometric design elements by substituting matched design criteria (see Section 2.4) associated with each automation-level scenario, and then determining and comparing effects of different automation levels on those elements.
Design elements considered in this paper include the required stopping sight distance, horizontal sight line offset, and length of crest and sag vertical curve.

3.1. Evaluation Scenarios. Based on basic driving automation levels $(L 0 \sim L 5)$ and the analysis in Section 2, six automation levels were considered: $L 0^{-}, L 0^{+}, L 1, L 2, L 3$, and $L 4 / 5$. Additional attention was paid to the ideal scenario of $L 3$, as L3 is the critical level where the engaged ADS initially performs the entire DDT. Some $L 3$ features are designed to automatically perform the DDT fallback and achieve a minimal risk condition in some circumstances, such as when an obstacle-free, adjacent shoulder is presented [14]. L3 with those features was defined as ideal $L 3$ in this paper. Because ideal $L 3$ with Lidars is equivalent to $L 4 / 5$, the scenario of ideal L3 only equipped with camera(s) and $\operatorname{radar}(\mathrm{s})$ was considered. It is easy to conclude that except for the height of the actor's sensing unit (with an upward divergence angle), values of all design criteria for ideal $L 3$ are the same as those for $L 4 / 5$. Therefore, seven analysis scenarios explored in this study are as follows: $\mathrm{LO}^{-}$(reference scenario referred to current geometric design practices), $L 0^{+}, L 1, L 2, L 3$, ideal $L 3$, and $L 4 / 5$.

3.2. Required Stopping Sight Distance. The required stopping sight distances (SSDs) of all scenarios except $L 3$ automation were computed as follows [25]:

$$
\mathrm{SSD}=0.278 \times V \times P b R T+\frac{V^{2}}{254\left[\left(A_{d} / 9.81\right) \pm i_{G}\right]},
$$

where $V(\mathrm{~km} / \mathrm{h})$ is the design speed; $i_{G}$ (rise/run, $\mathrm{m} / \mathrm{m}$ ) the longitudinal roadway grade. Take “-” on downgrades and “+” on upgrades.

Due to the preemptive braking during the TOT conducted by $L 3$ automation functions, the required SSD in $L 3$ can be written as follows:

$$
\begin{aligned}
S S D & =0.278 \times V \times P R T_{A D S}+\left[0.278 \times V \times T O T-\frac{1}{2}\left(\left(\frac{A_{d p}}{9.81}\right) \pm i_{G}\right) \times T O T^{2}\right]+\frac{\left[V-\left(\left(A_{d p} / 9.81\right) \pm i_{G}\right) \times T O T\right]^{2}}{254\left[\left(A_{d} / 9.81\right) \pm i_{G}\right]}, \\
& =0.278 \times V \times P b R T+\frac{\left[V-\left(\left(A_{d p} / 9.81\right) \pm i_{G}\right) \times T O T\right]^{2}}{254\left[\left(A_{d} / 9.81\right) \pm i_{G}\right]}-\frac{1}{2}\left(\left(\frac{A_{d p}}{9.81}\right) \pm i_{G}\right) \times T O T^{2},
\end{aligned}
$$

where $A_{d p}\left(\mathrm{~m} / \mathrm{s}^{2}\right), 2.5 \mathrm{~m} / \mathrm{s}^{2}$, is the preemptive deceleration rate activated by $L 3$ automation.

It should be noted that for all driving automation systems, the computed SSD should be within the perception range of sensors. Modern sensor systems (including camera, Radar, Lidar, etc.) allow for an environmental perception of up to $250 \mathrm{~m}$.
3.3. Horizontal Sight Line Offset. For the general safety design of the individual curve, the horizontal sight line offset (HSO) is commonly utilized to ensure that there is adequate sight distance on horizontal curves [71]. In order to study the effects of different automation levels on the sight distance across the inside of horizontal curves more conveniently, we only selected the typical horizontal sight line offset (HSO) 
where circular curves are longer than the sight distance for the pertinent $V$. Therefore, the derived values of $H S O$ proposed in [25] are:

$$
H S O=R\left[1-\cos \left(\frac{28.65 \times S S D}{R}\right)\right],
$$

where, $R(m)$ is the radius of the curve. It should be noted that the horizontal curve radius for the pertinent speed is determined from the superelevation rate (e) and side friction factor. The minimum radius is based on the threshold of driver/passenger comfort and that is sufficient to provide a margin of safety against skidding and vehicle rollover. Since most commercial ATs are realized by adding or refitting sensors and the onboard computer on the existing automobile model [72], there is no significant change in either vehicle size or mass. Thus, we assumed that $R$ in the context of AVs maintains the same value as that in the current practice.

Furthermore, given the $H S O$ and $R$, the required sight distance at the upper range for a speed can be inversely computed by (3), and then that maximum allowable speed can be obtained by (1) or (2).

\subsection{Lengths of Vertical Curves}

3.4.1. Crest Vertical Curves. Except for the $L 3$ scenario, the length of a crest vertical curve $\left(L_{c}\right)$ was derived as follows [25]:

$$
L_{c}= \begin{cases}\frac{\omega \times S S D^{2}}{100\left(\sqrt{2 h_{s}}+\sqrt{2 h_{o}}\right)^{2}}, & S S D<L_{c}, \\ 2 S S D-\frac{200\left(\sqrt{h_{s}}+\sqrt{h_{o}}\right)^{2}}{\omega}, & \text { SS } D>L_{c},\end{cases}
$$

where $\omega(\%)$ is the algebraic difference in grades. Note that minimum lengths of vertical curves should be larger than $0.6 \mathrm{~V}(\mathrm{~m})$ (also applies to lengths of sag vertical curves below) to approximate the range of current practice.

In the $L 3$ condition, the actor's sensing unit changes from the sensor (in $P R T_{\mathrm{ADS}}$ ) to the driver's eye (in TOT) during the stopping sight distance (in $P b R T$ ) as the considered worst-case scenario includes the take-over process from systems to human drivers. It is obvious that the human sight line plays a salient role in the value of $L_{c}$ due to lower eye height and slower response time. In order not to overestimate the computed $L_{c}$ value in $L 3$, it is necessary to adjust the (PbRT based) SSD in (4) to the TOT-based SSD, where $\quad($ TOT based SSD $)=S S D-0.278 \times V \times P R T_{A D S}$. Note that although the required SSD in either $L 1$ or $L 2$ also includes the distance within $P R T_{\text {automation }}(0.1 \mathrm{~s})$ except the human part, the overestimation in $L_{c}$ is not significant. Moreover, it seems to be reasonable not to separately consider SSD based on $P b R T_{\text {human }}$ because of the demanding immediate take-over actions by drivers who always engage in task 3 (Figure 1).

3.4.2. Sag Vertical Curves. From a practical point of view, three criteria besides $0.6 \mathrm{~V}$ were referred above for establishing the length of a sag vertical curve $\left(L_{s}\right)$ : sight distance at night or undercrossings, and passenger comfort were considered.

For sag vertical curves without an overhead vertical obstruction, $L_{s}$ of all scenarios apart from $L 3$ was derived as follows [25]:

$$
L_{s}= \begin{cases}\frac{\omega \times S S D^{2}}{200\left(h_{s}+S S D \tan \beta\right)}, & S S D<L_{s}, \\ 2 S S D-\frac{200\left(h_{s}+S S D \tan \beta\right)}{\omega}, & S S D>L_{s},\end{cases}
$$

where $\beta$ (deg) is the upward divergence angle of the sensing beam from the longitudinal axis of the vehicle. Note that passenger comfort should also be satisfied [25]:

$$
L_{s}=\frac{\omega \times V^{2}}{12.96 A_{c}}=\frac{\omega \times V^{2}}{395},
$$

where $A_{c}\left(\mathrm{~m} / \mathrm{s}^{2}\right)$, circa $0.3 \mathrm{~m} / \mathrm{s}^{2}$, is the centripetal acceleration for comfortable riding.

Another practical case where $L_{s}$ at grade separation structures was considered. The general equation for $L_{s}$ at undercrossings is [26]:

$$
L_{s}= \begin{cases}\frac{\omega \times S S D^{2}}{100\left[\sqrt{2\left(h_{c}-h_{s}\right)}+\sqrt{2\left(h_{c}-h_{o}\right)}\right]^{2}}, & \text { SS } D<L_{s}, \\ 2 S S D-\frac{400 h_{c}}{\omega}\left[1-\frac{h_{s}+h_{o}}{2 h_{c}}+\sqrt{\left(1-\frac{h_{s}}{h_{c}}\right) \times\left(1-\frac{h_{o}}{h_{c}}\right)}\right], & \text { SS } D>L_{s},\end{cases}
$$

where $h_{c}(m)$ is the vertical clearance. 
In the context of $L 3$ scenario, $L_{s}$ computing model ( (5)or (7) maintains the same revisions as $L_{c}$ (4), i.e., SSD in both $s$ (5) and (7) is replaced with the TOT-based SSD.

\section{Results}

Since current reliable operational design scenarios of ATs are mainly on high-type highways with high speeds and clear lane lines [73], the typical range of design speed from 60 to $100 \mathrm{~km} / \mathrm{h}$ was chosen.

4.1. Required Stopping Sight Distance. The typical $i_{G}$ value of 0.03 on both upgrade and downgrade was selected. The computed SSD for all scenarios on level roadways and grades were illustrated in Figure 3. As apparent from Figure 3, given the same $V$, the required SSDs of $L 1 \sim L 3$ increase and those of $L 0^{+}$, ideal $L 3$, and $L 4 / 5$ decrease at any grades compared with $L 0^{-}$. The required SSD in ideal $L 3$ and $L 4 / 5$ achieves the same smallest value, while that in $L 3$ is the longest. Hence, the order of the required SSD for all scenarios is: $L 3>L 2=L 1>L 0^{-}>L 0^{+}>$ideal $L 3=L 4 / 5$, in accordance with that of $P b R T$. Take the condition of $V=80 \mathrm{~km} / \mathrm{h}$ and $i_{G}=0$ as an example, change rates of the required SSD based on the reference value of $129.0 \mathrm{~m}\left(L 0^{-}\right)$are: $L 3(28 \%)$ $>L 2=L 1 \quad(19 \%)>L 0^{-}>L 0^{+} \quad(-15 \%)>$ ideal $\quad L 3=L 4 / 5$ $(-34 \%)$. Moreover, the absolute value of these change rates diminishes with the higher speed or on downgrade roadways.

4.2. Horizontal Sight Line Offset. Figures 4(a)-4(c) show the derived values of $H S O$ for all scenarios at the selected $V$ of 60 , 80 , and $100 \mathrm{~km} / \mathrm{h}$, respectively. For the curves shown in Figures 4(a)-4(c), the end of the solid line on the curve is the minimum radius where $e$ is equal to $12 \%$, while the dashed portion is the values less than the standard minimum radius for a maximum $e$ of $12 \%$. As depicted in Figures 3(a)-3(c), given the same $V$, the order of HSO for all scenarios at any radii exhibited very similar patterns with that of the required SSD, i.e., $L 3>L 2=L 1>L 0^{-}>L 0^{+}>$ideal $L 3=L 4 / 5$. For example, in the condition of $V=80 \mathrm{~km} / \mathrm{h}$ and $R=300 \mathrm{~m}$, change rates of $H S O$ based on the reference value of $6.9 \mathrm{~m}\left(L 0^{-}\right)$are: $L 3 \quad(63 \%)>L 2=L 1 \quad(41 \%)>L 0^{-}>L 0^{+}$ $(-28 \%)>$ ideal $L 3=L 4 / 5(-57 \%)$. Similarly, the amplitudes of these change rates shrink either with higher speed or larger radius.

Three cases of the given $H S O$ and $R$ were selected for analyzing possible impacts of driving automation levels on the maximum allowable speed (Figure 5): (1) Case I: $H S O=5 \mathrm{~m}$ and $R=175 \mathrm{~m}$; (2) Case II: $H S O=7 \mathrm{~m}$ and $R=280 \mathrm{~m}$; (3) Case III: $H S O=8 \mathrm{~m}$ and $R=492 \mathrm{~m}$. As can be seen in Figure 5, the order of the allowable speed is completely opposite to that of the required $H S O$, i.e., $L 4 / 5=$ ideal $L 3>L 0^{+}>L 0^{-}>L 1=L 2>L 3$. Take Case II as an example; change rates of the maximum allowable speed are: ideal $L 3$ and $L 4 / 5 \quad(26 \%)>L 0^{+} \quad(10 \%)>L 0^{-}>L 1=L 2 \quad(-11 \%)>L 3$ $(-16 \%)$.

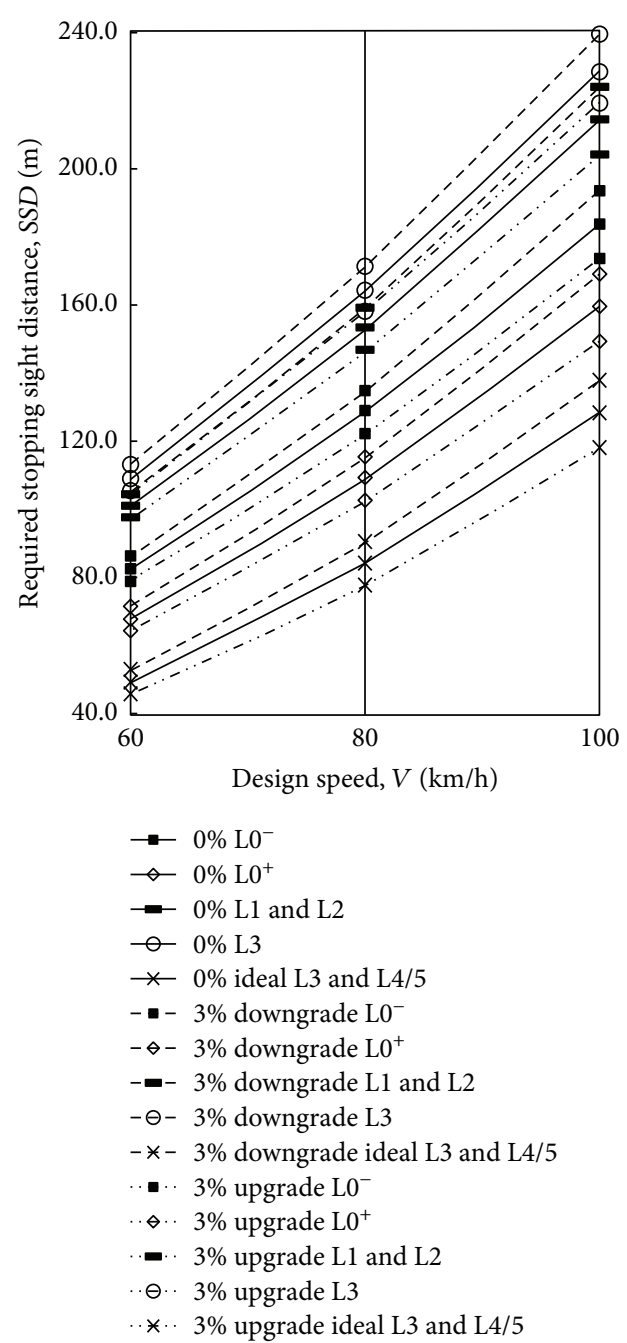

Figure 3: Required stopping sight distances for all scenarios on level roadways and grades.

\subsection{Lengths of Vertical Curves}

4.3.1. Crest Vertical Curves. The minimum $L_{c}$ for all scenarios at varied values of $\omega(0 \sim 16 \%)$ to provide the required SSD for the selected $V$ of 60,80 , and $100 \mathrm{~km} / \mathrm{h}$ was displayed in Figures 6(a)-6(c). For the curves (or lines) depicted in Figures 6(a)-6(c), the short-dashed curves, dash-dotted curves, and solid curves (or lines) indicate where $\mathrm{SSD}=L_{c}$, $\mathrm{SSD}>L_{c}$ and $\mathrm{SSD}<L_{c}$, respectively. As can be seen in Figures 6(a)-6(c), given the same $V$, the order of the minimum $L_{c}$ for all scenarios at any $\omega$ is basically consistent with that of the required SSD, i.e., $L 3 L 2=L 1 \geq L 0^{-} \geq L 0^{+} \geq$ideal $L 3 \geq L 4 / 5$. Note that the difference in the computed $L_{c}$ between $L 3$ and $L 1$ or $L 2$ is so marginal that it cannot be detected in Figures 6(a)-6(c), and $L_{c}$ computed by (4) in both ideal $L 3$ and $L 4 / 5$ at $V=60 \mathrm{~km} / \mathrm{h}$ is less than $0.6 \mathrm{~V}$ within the selected range of $\omega$. For example, in the condition of $V=80 \mathrm{~km} / \mathrm{h}$ and $\omega=8 \%$, change rates of $L_{c}$ based on the reference value of $125.7 \mathrm{~m}\left(L 0^{-}\right)$are: $L 3$ $(41.8 \%)>L 2=L 1 \quad(41.6 \%)>L 0^{-}>L 0^{+}(-28.1 \%)>$ ideal $L 3$ $(-59.1 \%)>L 4 / 5(-61.3 \%)$. For each $V$, the length of vertical 


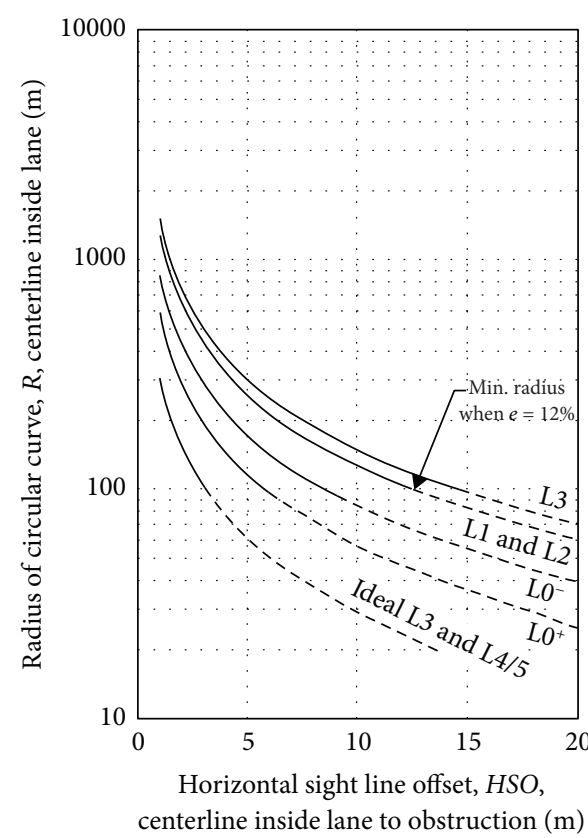

(a)

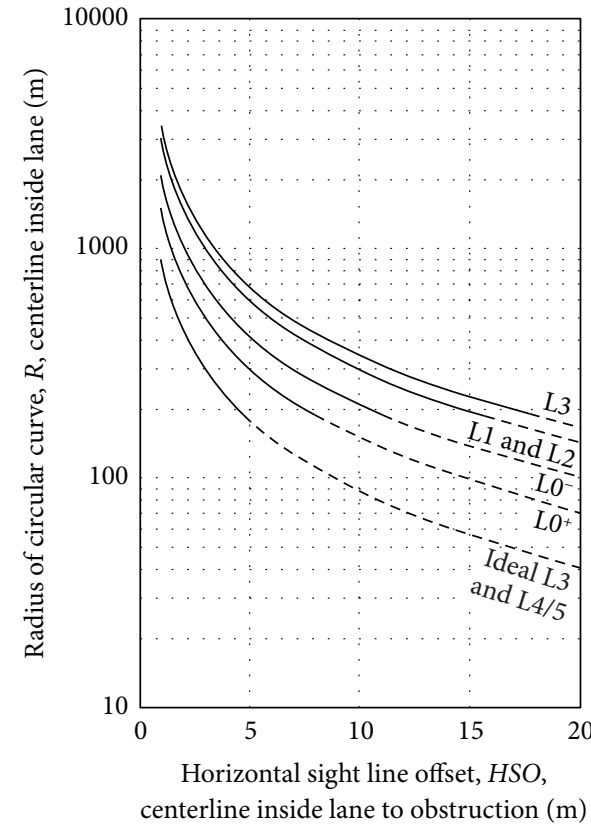

(b)

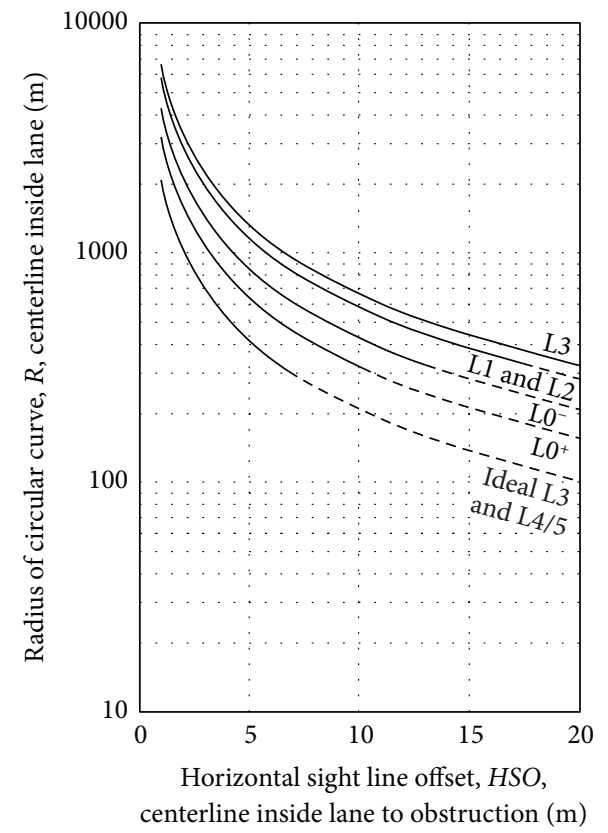

(c)

FIgURE 4: Required horizontal sight line offsets for all scenarios to provide stopping sight distances on horizontal curves: (a) $V=60 \mathrm{~km} / \mathrm{h}$; (b) $V=80 \mathrm{~km} / \mathrm{h}$; (c) $V=100 \mathrm{~km} / \mathrm{h}$.

curve per percent change in $\omega\left(K, L_{c}=K \times \omega\right)$ utilized to indicate the rate of vertical curvature [19] is a simple and convenient expression of the design control. Similarly, given the same $V$, the order of the rounded values of $K$ plotted in Figures 6(a)-6(c) is: $L 3=L 2=L 1>L^{-}>L^{+}>$ ideal $L 3 \geq L 4 / 5$.
4.3.2. Sag Vertical Curves. First, the minimum $L_{s}$ without an overhead vertical obstruction at varied values of $\omega(0 \sim 16 \%)$ to provide the required SSD for the selected $V$ of 60,80 , and $100 \mathrm{~km} / \mathrm{h}$ was depicted in Figures $7(\mathrm{a})-7(\mathrm{c})$. Similar to the context of $L_{c}$, the short-dashed curves, dash-dotted curves, and solid curves (or lines) in Figures $7(\mathrm{a})-7(\mathrm{c})$ indicate 


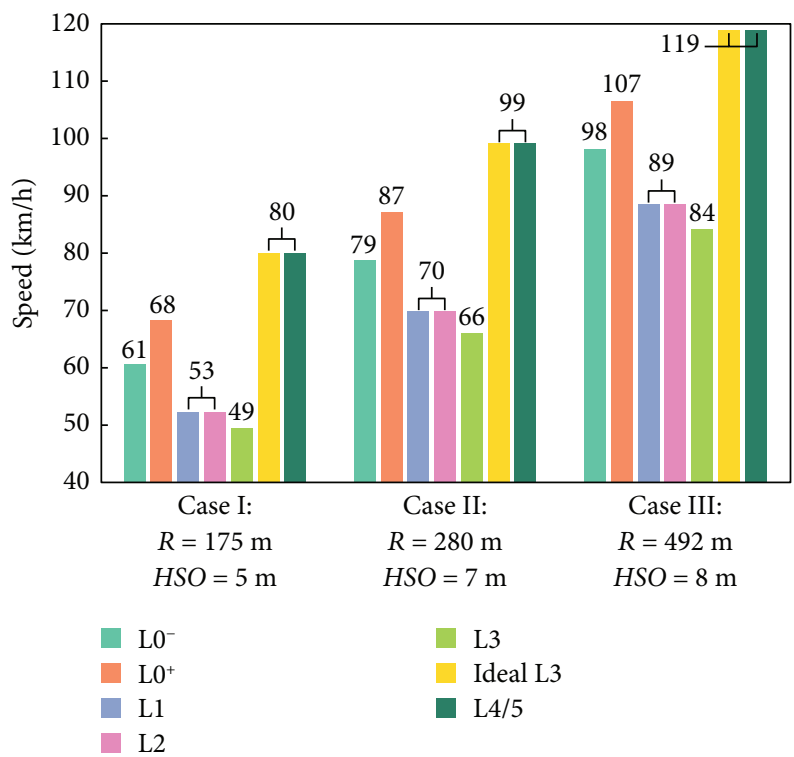

Figure 5: Comparison of maximum allowable speeds for all scenarios under the condition of given horizontal sight line offsets and radii.

where $\mathrm{SSD}=L_{s}, \mathrm{SSD}>L_{s}$ and $\mathrm{SSD}<L_{s}$, respectively. In addition, values of $L_{s}$ on the right of the red short-dashed line can satisfy the passenger comfort criterion. Given the same $V$, the order of the minimum $L_{s}$ at any $\omega$ is in line with that of $L_{c}$, i.e., $L 3 L 2=L 1 \geq L 0^{-} \geq L 0^{+} \geq$ideal $L 3 \geq L 4 / 5$. Furthermore, the order of the rounded values of $K$ is: $L 3=L 2=L 1>L 0^{-}>L 0^{+}>$ideal $L 3>L 4 / 5$. Take the condition of $V=80 \mathrm{~km} / \mathrm{h}$ and $\omega=8 \%$ as an example; change rates of $L_{s}$ based on the reference value of $145.3 \mathrm{~m}\left(L^{-}\right)$are: $L 3$ $(29.6 \%)>L 2=L 1 \quad(29.5 \%)>L 0^{-}>L 0^{+} \quad(-22.3 \%)>$ ideal $L 3=L 4 / 5(-67.0 \%)$. It should be noted that in the selected speed range, only $L_{s}$ in $L 0^{-}$and $L 1 \sim L 3$ could satisfy the comfort criterion at any $\omega$.

As a next step, for sag vertical curves at undercrossings with the typical $h_{c}$ value of $4.5 \mathrm{~m}$ [26], Figures $8(\mathrm{a})-8(\mathrm{c})$ show the minimum $L_{s}$ at varied $\omega(0 \sim 16 \%)$ to provide the required SSD for the selected $V$ of 60,80 , and $100 \mathrm{~km} / \mathrm{h}$. The criterion of passenger comfort was neglected because $L_{s}$ for all scenarios computed by (7) cannot meet that. Given the same $V$, the order of the minimum $L_{s}$ at any $\omega$ is $L 3 L 2=L 1 \geq L 0^{-} \geq L 0^{+} \geq L 4 / 5 \geq$ ideal $L 3$, and the order of the rounded values of $K$ is: $L 3=L 2=L 1>L 0^{-}>L 0^{+}>L 4 /$ $5>$ ideal $L 3$. Take the condition of $V=80 \mathrm{~km} / \mathrm{h}$ and $\omega=16 \%$ as an example; change rates of $L_{s}$ based on the reference value of $112.0 \mathrm{~m}\left(L 0^{-}\right)$are: $L 3(41.8 \%)>L 2=L 1 \quad(41.6 \%)$ $>L 0^{-}>L 0^{+}(-28.1 \%)>L 4 / 5(-53.4 \%)>$ ideal $L 3(-55.6 \%)$.

\section{Discussion}

The main intent of this study was to examine the impacts of varied driving automation levels on the geometric design of highways from the perspective of trucks. Particular attention was paid to explore differences in typical characteristics related to sight distances among varied levels (Section 2.3) and which design criteria of these characteristics seem reasonable and suitable for representing each level (Section 2.4). Finally, the influence of diverse automation levels with tailored features on the current models of geometric design elements (Section 3.2 3.4) and their computed values (Section 4) were analyzed. It should be noted that the derivation of our results mainly refers to the default values in specific geometry specifications $[25,26]$. Results based on other guidelines where inconsistent default values may be exhibited can be easily obtained and examined by the methodology in this study. It can be anticipated that the results of this paper are sound since the differences between those values are generally small. Also, those automation features considered in this study do not include limited capacities of perception sensors in adverse weather conditions (e.g., rain or fog), i.e., the road environment for all evaluation scenarios is ideal.

There are interesting insights, both qualitative and quantitative, to be gleaned from this study. Firstly, characteristics with respect to sight distances consisting of $P b R T$ and $h_{s}$ (with $\beta$ ) are the main and explicit differences among manifold automation levels. Previous studies [10,12] have investigated those characteristics in the context of highly automated driving. We extended previous findings to all driving automation scopes $(L 0 \sim L 5)$ supported by a broad body of literature. Notably, decomposed components of $P b R T$ for each level were established. The $P R T_{\text {automation }}$ and the $P R T_{\mathrm{ADS}}$ followed by TOT were introduced into the $P b R T$ structure in $L 1 / L 2$ and $L 3$, respectively, where the control right of ATs is shared between the human driver and systems to some extent. Furthermore, sensing actors for all levels were reconsidered and adjusted in the worst-case 


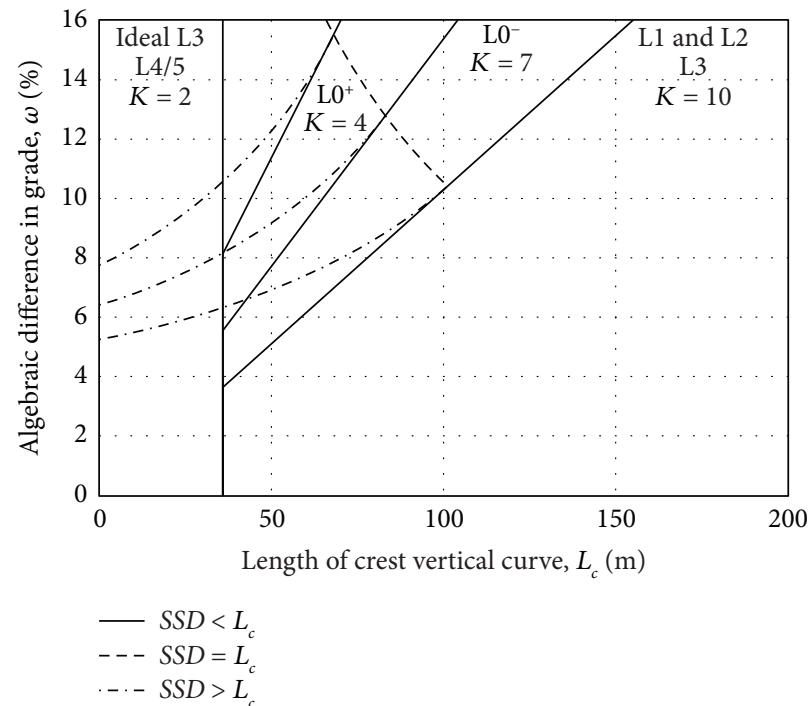

(a)

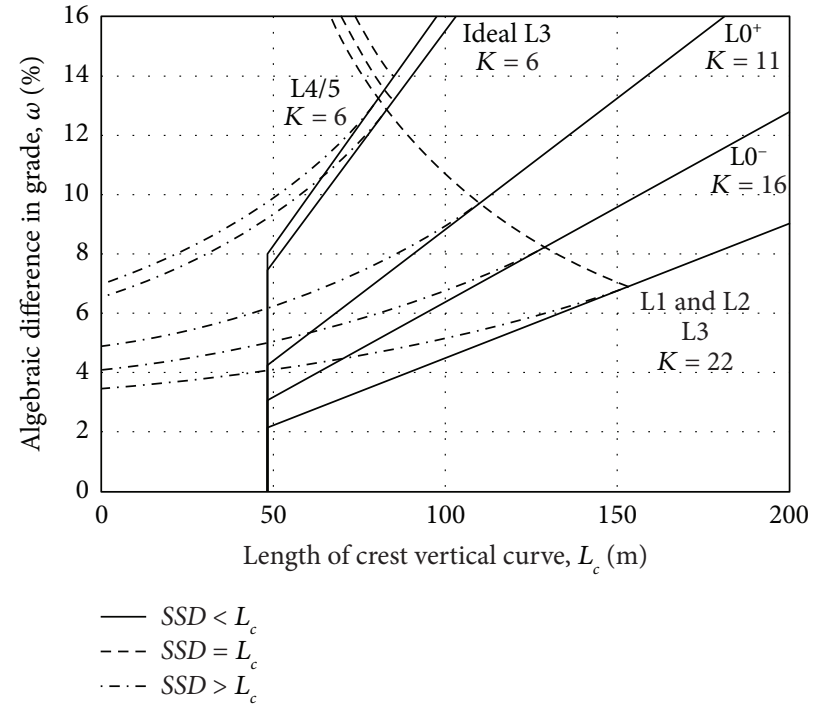

(b)

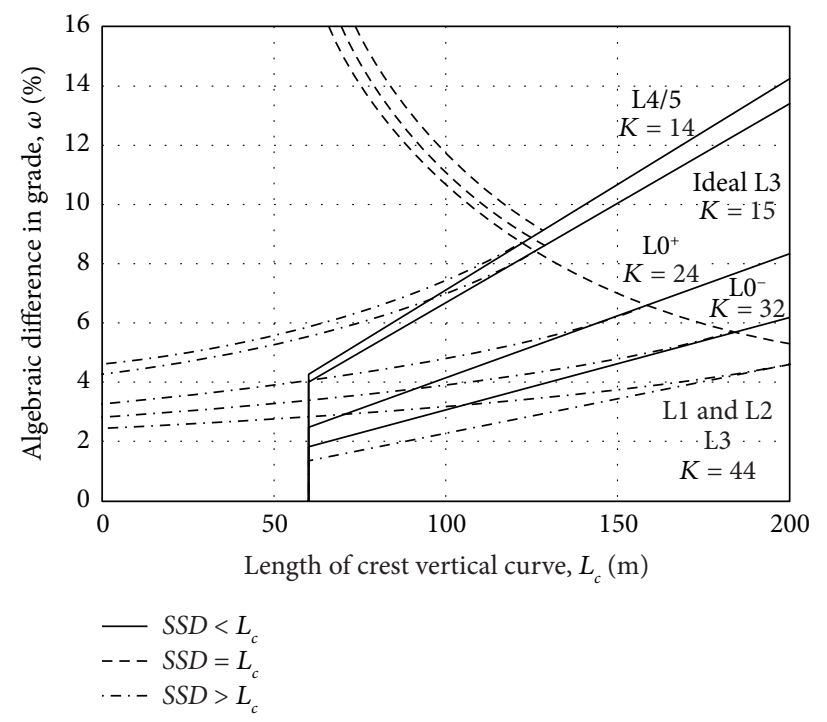

(c)

Figure 6: Design controls for crest vertical curves for all scenarios: (a) $V=60 \mathrm{~km} / \mathrm{h}$; (b) $V=80 \mathrm{~km} / \mathrm{h}$; (c) $V=100 \mathrm{~km} / \mathrm{h}$.

scenario. In short, features of perception sensors and system functions will replace those of humans gradually in defining characteristics of sight distances.

Another important finding of this study is that with the advancement of automation levels, requirements of geometric design elements including the required SSD, HSO, and the minimum $L_{c}$ and $L_{s}$ moderate initially $\left(L 0^{+}\right)$, then develop toward the strict direction ( $L 1 \sim L 3)$, and finally diminish significantly $(L 4 / 5)$ compared with those in $L 0^{-}$, for e.g., longer required SSD is necessitated in $L 1 \sim L 3$ and smaller $L_{c}$ in $L 0^{+}$or $L 4 / 5$. In other words, both $L 0^{+}$and $L 4 / 5$ (especially the latter) can fully adapt to the geometry condition of highways in service, while $L 1 \sim L 3$ (especially $L 3$ ) need higher-type geometric specifications to ensure safety [73]. The explanation for this finding could be that quick $P b R T$ or/and high $h_{s}$ (with large $\beta$ ) are achieved in $L 0^{-}$and $L 4 / 5$, while the slower $P b R T_{\text {human }}$ or TOT and the continued request of drivers in the control loop deteriorate the anticipated performance of automation systems in $L 1 \sim L 3$.

This study also found that larger maximum allowable speeds can be obtained in both $L 0^{-}$and $L 4 / 5$ (especially the latter) but smaller in $L 1 \sim L 3$ (especially $L 3$ ) given the $H S O$ and $R$. The latter (smaller allowable speeds in $L 1 \sim L 3$ ) can be verified by Garcia et al. [74] through a real semiautonomous vehicle test along highways. A more interesting finding is that the requirements of all considered design elements in 


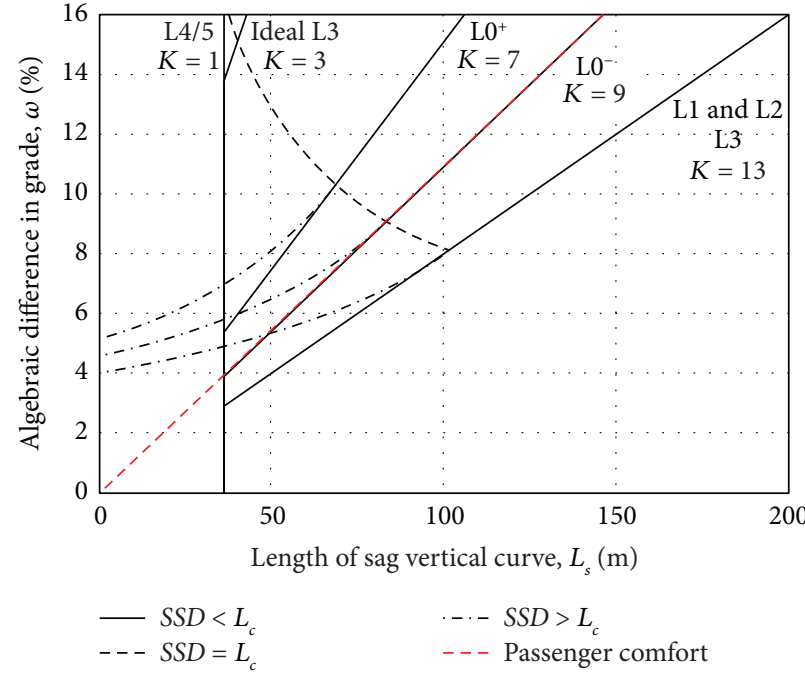

(a)

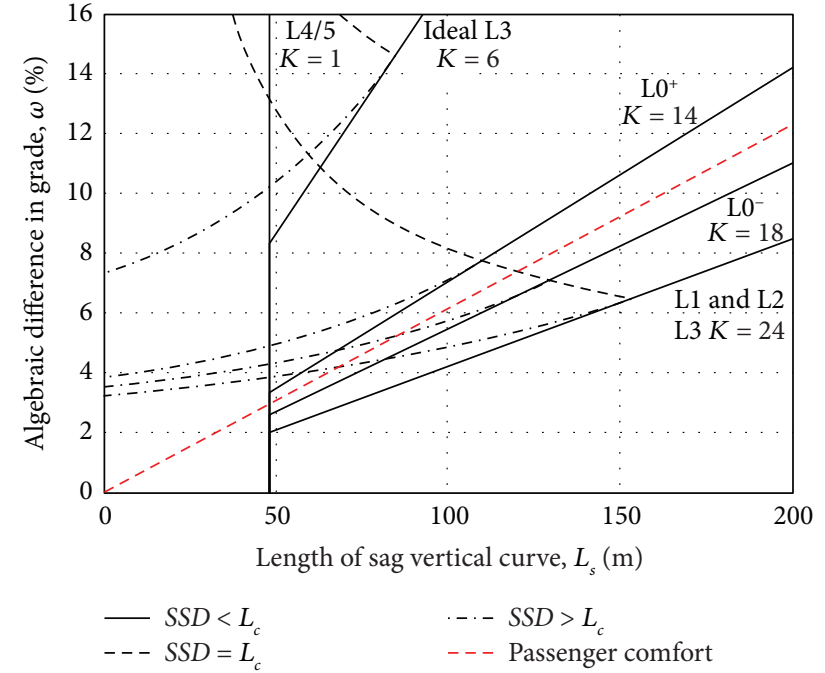

(b)

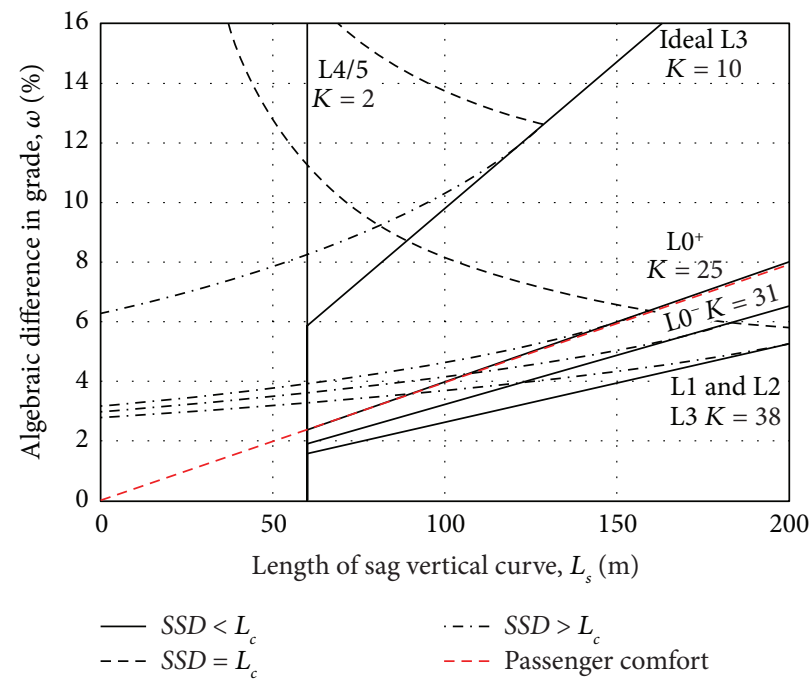

(c)

Figure 7: Design controls for sag vertical curves on open road conditions for all scenarios: (a) $V=60 \mathrm{~km} / \mathrm{h}$; (b) $V=80 \mathrm{~km} / \mathrm{h}$; (c) $V=100 \mathrm{~km} / \mathrm{h}$.

ideal $L 3$ are basically the same as those in $L 4 / 5$ except for requirements of sag vertical curves. Lower $h_{s}$ (with narrower $\beta$ ) enables ideal $L 3$ to acquire longer $L_{s}$ on open road conditions than $L 4 / 5$, however, somewhat shorter $L_{s}$ at undercrossings.

Some limitations of the present study must be considered. Obviously, a single value selected for each characteristic such as $P b R T$ or $h_{s}$ cannot provide a complete description of it. More validations and research have to be conducted before definitive conclusions of design criteria for all automation levels can be drawn. However, even at this stage, the design criteria estimated in this article seem to be in a reasonable agreement with the data exhibited in published studies and released products. It seems reasonable to expect that this approach will help to solve practical problems such as the geometric design of highways more economically and conveniently. Also, this study was conducted from the perspective of trucks, which covers only one class of design vehicles. The passenger car is definitely one of the most representative vehicle types for on-road motor vehicles equipped with driving automation systems and for purposes of geometric highway design. Results in the context of passenger cars can be easily obtained and examined by the methodology in this study. Another limitation of this study is that the typical operation form of ATs, platoon, or roadtrains is not considered. Although one single truck in this study can be regarded as the leader in the platoon, further research is need on actual longitudinal and lateral driving behaviors of followers under a real road environment. Finally, this study only estimated the sight distance on the horizontal or vertical plane and overlooked the threedimensional (3D) nature of the highway infrastructure. The authors have proposed an automatic framework for 3D highway sight distance analysis in [71]. Detailed impact analysis of automation systems on sight distance along the 3D highway alignment is expected in the following work. 


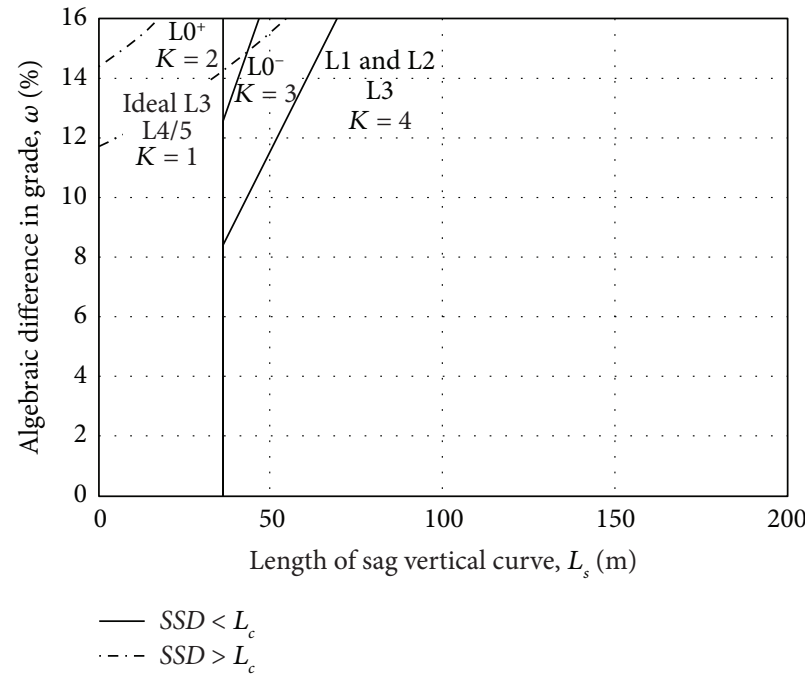

(a)

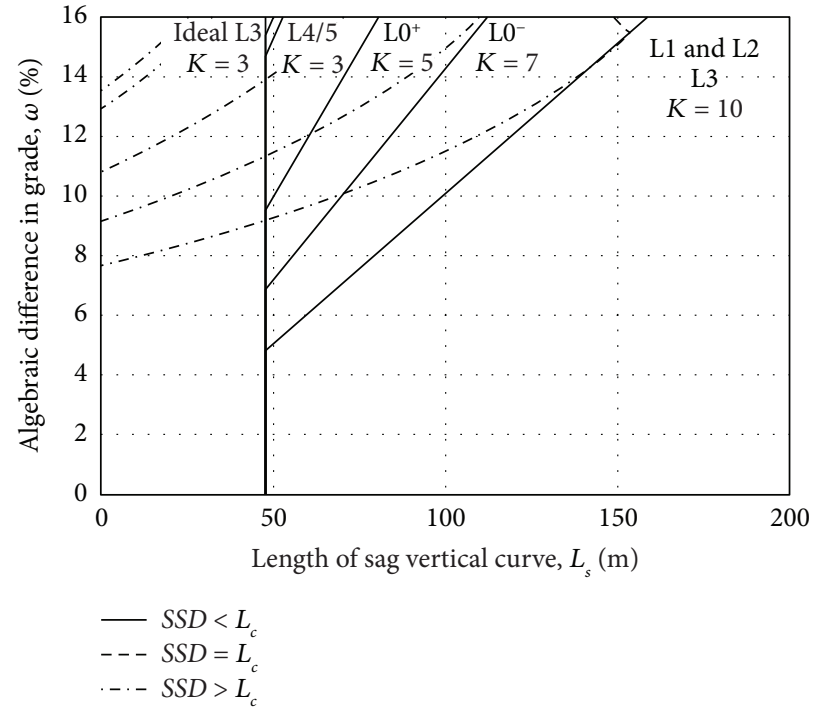

(b)

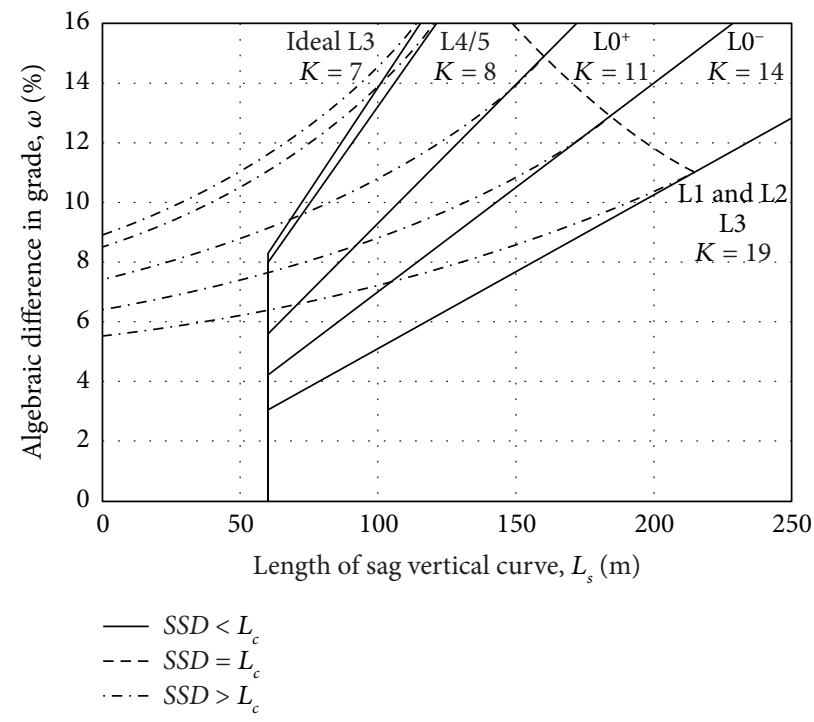

(c)

Figure 8: Design controls for sag vertical curves at undercrossings for all scenarios: (a) $V=60 \mathrm{~km} / \mathrm{h}$; (b) $V=80 \mathrm{~km} / \mathrm{h}$; (c) $V=100 \mathrm{~km} / \mathrm{h}$.

\section{Conclusions}

The results presented here offer new insights into differences in typical characteristics related to sight distances among varied driving automation levels and possible positive or negative effects of those characteristics on geometric highway design. Results show that high or full driving automation could substantially lower the requirements of geometric design, which is consistent with previous findings. Active safety systems have a similar role but with moderate effects. Differently, the driver assistance and partial or conditional automation systems put a higher demand on the road geometric design from the safety point of view.

Primary implications of this study are expected to have design consequences for the real-world geometry of dedicated lanes. Also, our findings can be used to facilitate the identification of potential risk sections on highways in service for the operation of $L 1 \sim L 3$ and otherwise provide a methodological basis for the formulation of speed limit policy. Furthermore, this paper may be useful in urging researchers or technicians of automated driving systems to take into account driver states and performances in the takeover process.

\section{Data Availability}

The data used to support the findings of this study are available from the corresponding author upon request.

\section{Conflicts of Interest}

The authors declare that they have no conflicts of interest. 


\section{Acknowledgments}

This study was supported by the Postgraduate Research \& Practice Innovation Program of Jiangsu Province under grant no. KYCX18_0152, the National Natural Science Foundation of China under grant no. 51878163, China Scholarship Council, and National Demonstration Center for Experimental Road and Traffic Engineering Education (Southeast University).

\section{References}

[1] N. Virdi, H. Grzybowska, S. T. Waller, and V. Dixit, "A safety assessment of mixed fleets with connected and autonomous vehicles using the surrogate safety assessment module," Accident Analysis \& Prevention, vol. 131, pp. 95-111, 2019.

[2] M. Amirgholy, M. Shahabi, and H. Oliver Gao, "Traffic automation and lane management for communicant, autonomous, and human-driven vehicles," Transportation Research Part C: Emerging Technologies, vol. 111, pp. 477-495, 2020.

[3] A. Alam, B. Besselink, V. Turri, J. Martensson, and K. H. Johansson, "Heavy-duty vehicle platooning for sustainable freight transportation a cooperative method to enhance safety and efficiency," IEEE Control Systems Magazine, vol. 35, no. 6, pp. 34-56, 2015.

[4] N. A. Stanton, M. Young, and B. McCaulder, "Drive-by-wire: the case of driver workload and reclaiming control with adaptive cruise control," Safety Science, vol. 27, no. 2-3, pp. 149-159, 1997.

[5] M. M. Hoque, Q. Lu, and C. Xin, "Effect of highway lane management policy of heavy vehicles on the cost of flexible pavement," Transportation Engineering Part A: Systems, vol. 144, no. 11, Article ID 04018072, 2018.

[6] M. Klumpp and H. Zijm, "Logistics innovation and social sustainability: how to prevent an artificial divide in humancomputer interaction," Journal of Business Logistics, vol. 40, no. 3, pp. 265-278, 2019.

[7] V. Ardalan and S. Antonio, "Energy saving potentials of connected and automated vehicles," Transportation Research Part C: Emerging Technologies, vol. 95, pp. 822-843, 2018.

[8] K. J. Bucklew, "Improving freight roadway transportation with dedicated truck lanes: opportunities and issues," Transportation Journal, vol. 50, no. 4, pp. 431-445, 2011.

[9] S. S. Washburn and L. D. Washburn, Future Highways Automated Vehicles, SunCam, Wellington, FL, USA, 2018.

[10] P. Intini, P. Colonna, N. Berloco, V. Ranieri, and Washburn, "Rethinking the main road design concepts for future automated vehicles native roads," European Transport-Trasporti Europei, no. 73, Article ID 3, 2019.

[11] N. E. Thomas and F. J. Martinez-Perez, "Impacts of roadtrains on the geometric design of highways," Transportation Engineering, vol. 141, no. 4, Article ID 04014087, 2015.

[12] J. Khoury, K. Amine, and R. A. Saad, "An initial investigation of the effects of a fully automated vehicle fleet on geometric design," Journal of Advanced Transportation, vol. 2019, Article ID 6126408, 10 pages, 2019.

[13] Ministry of Transport of the People's Republic of China, Statistics and Development Bulletin of the Transportation Industry in 2016, China Communications Press, Beijing, China, 2017.

[14] Society of Automotive Engineers (SAE) International, Taxonomy and Definitions for Terms Related to Driving Automation Systems for on-Road Motor Vehicles: J3016, Society of
Automotive Engineers (SAE) International, Warrendale, PA, USA, 2018.

[15] D. Chen, A. Srivastava, S. Ahn, and T. Li, "Traffic dynamics under speed disturbance in mixed traffic with automated and non-automated vehicles," Transportation Research Part C: Emerging Technologies, vol. 113, pp. 293-313, 2020.

[16] D.-F. Xie, X.-M. Zhao, and Z. He, "Heterogeneous traffic mixing regular and connected vehicles: modeling and stabilization," IEEE Transactions on Intelligent Transportation Systems, vol. 20, no. 6, pp. 2060-2071, 2019.

[17] Y. Wang, L. Wei, and P. Chen, “Trajectory reconstruction for freeway traffic mixed with human-driven vehicles and connected and automated vehicles," Transportation Research Part C: Emerging Technologies, vol. 111, pp. 135-155, 2020.

[18] R. Parasuraman, T. B. Sheridan, and C. D. Wickens, "Situation awareness, mental workload, and trust in automation: viable, empirically supported cognitive engineering constructs," Journal of Cognitive Engineering and Decision Making, vol. 2, no. 2, pp. 140-160, 2008.

[19] M. S. Young and N. A. Stanton, "What's skill got to do with it? Vehicle automation and driver mental workload," Ergonomics, vol. 50, no. 8, pp. 1324-1339, 2007.

[20] A. H. Jamson, N. Merat, O. M. J. Carsten, and F. C. H. Lai, "Behavioural changes in drivers experiencing highly-automated vehicle control in varying traffic conditions," Transportation Research Part C: Emerging Technologies, vol. 30, pp. 116-125, 2013.

[21] Z. Zhang, J. Li, Y. Guo, C. Yang, and C. Wang, "3D highway curve reconstruction from mobile laser scanning point clouds," IEEE Transactions on Intelligent Transportation Systems, vol. 21, no. 11, pp. 4762-4772, 2020.

[22] D. Shin, K. M. Park, and M. Park, "High definition map-based localization using ADAS environment sensors for application to automated driving vehicles," Applied Sciences-Basel, vol. 10, no. 14, Article ID 4924, 2020.

[23] M. Berk, O. Schubert, H.-M. Kroll, B. Buschardt, and D. Straub, "Exploiting redundancy for reliability analysis of sensor perception in automated driving vehicles," IEEE Transactions on Intelligent Transportation Systems, vol. 21, no. 12 , pp. 5073-5085, 2020.

[24] S. Jing, X. Zhao, F. Hui, A. J. Khattak, and L. Yang, "Cooperative CAVs optimal trajectory planning for collision avoidance and merging in the weaving section," Transportmetrica B: Transport Dynamics, vol. 9, no. 1, pp. 219-236, 2021.

[25] American Association of State Highway and Transportation Officials (AASHTO), A Policy on Geometric Design of Highways and Streets, AASHTO, Washington, DC, USA, 7th edition, 2018.

[26] Ministry of Transport of the People's Republic of China, "Design Specification for Highway Alignment," JTG D20-2017, China Communications Press, Beijing, China, 2017.

[27] X. Wu, L. N. Boyle, D. Marshall, and W. O’Brien, "The effectiveness of auditory forward collision warning alerts," Transportation Research Part F: Traffic Psychology and Behaviour, vol. 59, pp. 164-178, 2018.

[28] M. L. Aust, J. Viström, and M. Vistrom, "Effects of forward collision warning and repeated event exposure on emergency braking," Transportation Research Part F: Traffic Psychology and Behaviour, vol. 18, pp. 34-46, 2013.

[29] J. G. Gaspar, C. W. Schwarz, T. L. Brown, and J. Kang, "Gaze position modulates the effectiveness of forward collision warnings for drowsy drivers," Accident Analysis \& Prevention, vol. 126, pp. 25-30, 2019. 
[30] N. B. Sarter and D. D. Woods, "Situation awareness: a critical but ill-defined phenomenon," The International Journal of Aviation Psychology, vol. 1, no. 1, pp. 45-57, 1991.

[31] N. A. Stanton and M. S. Young, "A proposed psychological model of driving automation," Theoretical Issues in Ergonomics Science, vol. 1, no. 4, pp. 315-331, 2000.

[32] N. Merat, A. H. Jamson, F. C. H. Lai, and O. Carsten, "Highly automated driving, secondary task performance, and driver state," Human Factors: The Journal of the Human Factors and Ergonomics Society, vol. 54, no. 5, pp. 762-771, 2012.

[33] J. C. F. de Winter, R. Happee, M. H. Martens, and N. A. Stanton, "Effects of adaptive cruise control and highly automated driving on workload and situation awareness: a review of the empirical evidence," Transportation Research Part F: Traffic Psychology and Behaviour, vol. 27, pp. 196-217, 2014.

[34] H. Clark and J. Feng, "Age differences in the takeover of vehicle control and engagement in non-driving-related activities in simulated driving with conditional automation," vol. 106, pp. 468-479, 2015.

[35] A. O. Ferdinand and N. Menachemi, "Associations between driving performance and engaging in secondary tasks: a systematic review," American Journal of Public Health, vol. 104, no. 3, pp. E39-E48, 2014.

[36] N. Strand, J. Nilsson, I. C. M. Karlsson, and L. Nilsson, "Semi-automated versus highly automated driving in critical situations caused by automation failures," Transportation Research Part F: Traffic Psychology and Behaviour, vol. 27, pp. 218-228, 2014.

[37] K. L. Young, P. M. Salmon, and M. Cornelissen, "Missing links? The effects of distraction on driver situation awareness," Safety Science, vol. 56, pp. 36-43, 2013.

[38] M. Green, “"How long does it take to stop?” methodological analysis of driver perception-brake times," Transportation Human Factors, vol. 2, no. 3, pp. 195-216, 2000.

[39] H. Winner, K. Winter, B. Lucas et al., ACC Adaptive Cruise Control," Plochingen, Robert Bosch Gmbh, German, 2003.

[40] A. Sarker, H. Shen, M. Rahman et al., "A review of sensing and communication, human factors, and controller aspects for information-aware connected and automated vehicles," IEEE Transactions on Intelligent Transportation Systems, vol. 21, no. 1, pp. 7-29, 2020.

[41] K. Zeeb, A. Buchner, and M. Schrauf, "What determines the take-over time? An integrated model approach of driver takeover after automated driving," Accident Analysis \& Prevention, vol. 78, pp. 212-221, 2015.

[42] D. Gonzalez, J. Perez, V. Milanes, and F. Nashashibi, "A review of motion planning techniques for automated vehicles," IEEE Transactions on Intelligent Transportation Systems, vol. 17, no. 4, pp. 1135-1145, 2016.

[43] D. D. Salvucci and N. A. Taatgen, "Threaded cognition: an integrated theory of concurrent multitasking," Psychological Review, vol. 115, no. 1, pp. 101-130, 2008.

[44] B. Zhang, J. de Winter, S. Varotto, R. Happee, and M. Martens, "Determinants of take-over time from automated driving: a meta-analysis of 129 studies," Transportation Research Part F: Traffic Psychology and Behaviour, vol. 64, pp. 285-307, 2019.

[45] C. Gold, D. Damböck, L. Lorenz, and K. Bengler, “"Take over!" How long does it take to get the driver back into the loop?," Proceedings of the Human Factors and Ergonomics Society Annual Meeting, vol. 57, no. 1, pp. 1938-1942, 2013.

[46] K. Zeeb, A. Buchner, and M. Schrauf, "Is take-over time all that matters? The impact of visual-cognitive load on driver take-over quality after conditionally automated driving," Accident Analysis \& Prevention, vol. 92, pp. 230-239, 2016.

[47] G. Markkula, J. Engström, J. Lodin, J. Bärgman, and T. Victor, "A farewell to brake reaction times? Kinematics-dependent brake response in naturalistic rear-end emergencies," Accident Analysis \& Prevention, vol. 95, pp. 209-226, 2016.

[48] E. Yurtsever, J. Lambert, A. Carballo, and K. Takeda, "A survey of autonomous driving: common practices and emerging technologies," IEEE Access, vol. 8, pp. 58443-58469, 2020.

[49] E. Marti, J. Perez, M. A. de Miguel, and F. Garcia, "A review of sensor technologies for perception in automated driving," IEEE Intelligent Transportation Systems Magazine, vol. 11, no. 4, pp. 94-108, 2015.

[50] J. Van Brummelen, M. O’Brien, D. Gruyer, and H. Najjaran, "Autonomous vehicle perception: the technology of today and tomorrow," Transportation Research Part C: Emerging Technologies, vol. 89, pp. 384-406, 2018.

[51] F. Rosique, P. J. Navarro, C. Fernandez, and A. Padilla, "A systematic review of perception system and simulators for autonomous vehicles research," Sensors, vol. 19, no. 3, Article ID $648,2019$.

[52] H. Noorvand, G. Karnati, and B. S. Underwood, "Autonomous vehicles," Transportation Research Record: Journal of the Transportation Research Board, vol. 2640, no. 1, pp. 21-28, 2017.

[53] K. Yoneda, N. Suganuma, R. Yanase, and M. Aldibaja, "Automated driving recognition technologies for adverse weather conditions," IATSS Research, vol. 43, no. 4, pp. 253-262, 2019.

[54] A. Lotz, N. Russwinkel, and E. Wohlfarth, "Response times and gaze behavior of truck drivers in time critical conditional automated driving take-overs," Transportation Research Part F: Traffic Psychology and Behaviour, vol. 64, pp. 532-551, 2019.

[55] S. Bao, D. J. LeBlanc, J. R. Sayer, and C. Flannagan, "Heavytruck drivers' following behavior with intervention of an integrated, in-vehicle crash warning system," Human Factors: The Journal of the Human Factors and Ergonomics Society, vol. 54, no. 5, pp. 687-697, 2012.

[56] Z. H. Sun, G. Bebis, and R. Miller, "Heavy-truck drivers' following behavior with intervention of an integrated, invehicle crash warning system: a field evaluation," IEEE Transactions on Pattern Analysis and Machine Intelligence, vol. 28, no. 5, pp. 694-711, 2006.

[57] M. S. Young and N. A. Stanton, "Back to the future: brake reaction times for manual and automated vehicles," Ergonomics, vol. 50, no. 1, pp. 46-58, 2007.

[58] R. Parasuraman, R. Molloy, and I. L. Singh, "Performance consequences of automation-induced "complacency"," The International Journal of Aviation Psychology, vol. 3, no. 1, pp. 1-23, 1993.

[59] A. F. L. Larsson, K. Kircher, and J. Andersson Hultgren, "Learning from experience: familiarity with ACC and responding to a cut-in situation in automated driving," Transportation Research Part F: Traffic Psychology and Behaviour, vol. 27, pp. 229-237, 2014.

[60] N. A. Stanton, M. S. Young, G. H. Walker, H. Turner, and S. Randle, "Automating the driver's control tasks," The International Journal of Aviation Psychology, vol. 5, no. 3, pp. 221-236, 2010.

[61] F. Naujoks, C. Purucker, K. Wiedemann, A. Neukum, S. Wolter, and R. Steiger, "Driving performance at lateral system limits during partially automated driving," Accident Analysis \& Prevention, vol. 108, pp. 147-162, 2017. 
[62] F. Naujoks, S. Höfling, C. Purucker, and K. Zeeb, "From partial and high automation to manual driving: relationship between non-driving related tasks, drowsiness and take-over performance," Accident Analysis \& Prevention, vol. 121, pp. 28-42, 2018.

[63] A. DinparastDjadid, J. D. Lee, J. Domeyer et al., "Designing for the extremes: modeling drivers' response time to take back control from automation using bayesian quantile regression," Human Factors, Article ID 0018720819893429, 2019.

[64] D. K. Fambro, K. Fitzpatrick, and R. J. Koppa, Determination of Stopping Sight Distances: National Cooperative Highway Research Program (NCHRP) Report 400, Transportation Research Board, Washington, DC, USA, 1997.

[65] H. Wang, B. Wang, B. Liu, X. Meng, and G. Yang, "Pedestrian recognition and tracking using $3 \mathrm{D}$ LiDAR for autonomous vehicle," Robotics and Autonomous Systems, vol. 88, pp. 71-78, 2017.

[66] Wevolver, 2020 Autonomous Vehicle Technology Report, Wevolver, Amsterdam, The Netherlands, 2020.

[67] B. S. Jahromi, T. Tulabandhula, and S. Cetin, "Real-time hybrid multi-sensor fusion framework for perception in autonomous vehicles," Sensors, vol. 19, no. 20, Article ID 4357, 2019.

[68] X. Y. Yu and M. Marinov, "Real-time hybrid multi-sensor fusion framework for perception in autonomous vehicles," Sustainability, vol. 12, no. 8, Article ID 3281, 2020.

[69] C. Alexander, L. Jacob, M. C. Abraham et al., "LIBRE: The Multiple 3D LiDAR Dataset," 2003, https://arxiv.org/abs/ 2003.06129.

[70] W. Buller, B. Wilson, J. Garbarino et al., Radar Congestion Study, Report No. DOT HS 812 632, National Highway Traffic Safety Administration (NHTSA), Washington, DC, USA, 2018.

[71] Y. Ma, S. Easa, J. Cheng, and B. Yu, "Automatic framework for detecting obstacles restricting 3D highway sight distance using MLS data," Journal of Computing in Civil Engineering, In press, 2021.

[72] M. A. Walte, S. Oscar, L. Régis, H. J. Hadj, and O. M. Cristina, "Automated driving: a literature review of the take over request in conditional automation," Electronics, vol. 9, no. 12, Article ID 2087, 2020.

[73] A. Garcia and F. J. Camacho-Torregrosa, "Influence of lane width on semi-autonomous vehicle performance," Transportation Research Records, vol. 2674, no. 9, pp. 279-286, Article ID 0361198120928351, 2020.

[74] A. Garcia, F. J. Camacho-Torregrosa, and P. V. P. Baez, "Examining the effect of road horizontal alignment on the speed of semi-automated vehicles," Accident Analysis \& Prevention, vol. 146, Article ID 105732, 2020. 